\title{
Dual-UEGO Active Catalyst Control for Emissions Reduction: Design and Experimental Validation
}

\author{
Giovanni Fiengo, J. W. Grizzle, Jeffrey A. Cook, and Amey Y. Karnik
}

\begin{abstract}
This paper investigates active control of an aftertreatment system for a conventional spark ignition engine equipped with one or two three-way catalysts and two oxygen sensors. The control objective is to maximize the simultaneous conversion efficiencies of oxides of nitrogen and unburned hydrocarbons. Linear exhaust gas oxygen sensors are used to measure air-fuel ratio upstream and downstream of each catalyst. A series controller configuration is adopted. The upstream controller provides relatively rapid response to disturbances on the basis of measured feedgas air-fuel ratio, while the downstream controller uses the feedgas and post-catalyst air-fuel ratio measurements to compensate for the bias corrupting the feedgas air-fuel ratio measurement. The performance and robustness of the proposed control system in the face of noise and model uncertainty are first evaluated through extensive simulations. The control strategy is then experimentally verified in a dynamometer test cell and its performance compared with an existing proprietary controller that is based on the more common switching-type air-fuel ratio sensors.
\end{abstract}

\section{INTRODUCTION}

Conventional automotive gasoline engines (that is, homogeneous charge, stoichiometric air-fuel ratio $(A / F)$ ), employ a three-way catalytic converter (TWC) to oxidize $H C$ and $C O$ emissions and reduce $N O x$. Traditionally, the control emphasis has been on $A / F$ feedback using an exhaust gas oxygen (EGO) sensor located in the exhaust manifold upstream of the TWC to maintain the $A / F$ near stoichiometry and achieve high simultaneous conversion efficiencies. Recent requirements for on board diagnostics (OBD) have mandated an additional EGO sensor downstream of the TWC, and this secondary sensor is often used to trim the control action of the primary sensor; see [13] for related work using switching-type (HEGO) sensors and [1], [16] for work using linear, Universal Exhaust Gas Oxygen (UEGO) sensors.

The goal of this paper is to present an active catalyst control strategy ${ }^{1}$ based on the use of linear exhaust gas oxygen (UEGO) sensors both upstream and downstream of the catalyst. The development starts with phenomenological models of the engine, catalytic convertor, and sensor set. The sensor model captures a very important phenomenon, namely that upstream of the catalyst, non-equilibrium effects in the exhaust gas result in a bias error in the air-fuel ratio measurement. The bias typically results in the measured air-fuel ratio value being richer than the actual air-fuel ratio, causing the control system to adjust to an air-fuel ratio that is lean of stoichiometry. This is turn results in degraded NOx emissions. The phenomenological models are used to develop a control strategy that specifically addresses the bias error in the air-fuel ratio sensor upstream of the catalyst. The values of the control parameters are determined on the basis of nonlinear optimization of catalyst conversion efficiency, subject to drivability constraints. The phenomenological models are also used in a detailed closed-loop robustness analysis.

Experimental verification of the control strategy's performance is reported for two dynamometer test cells. One cell is equipped with a $5.4 L \mathrm{~V} 8$-engine and the other with a $2.3 \mathrm{~L}$ in-line four-cylinder engine. The obtained results are remarkably consistent with the results reported in the simulation analysis.

\section{System Model}

Figure 1 illustrates the nominal system to be controlled. It consists of a Spark Ignition Internal Combustion Engine (SI-ICE) equipped with a catalytic convertor. Universal Exhaust gas oxygen (UEGO) sensors provide a measurement of the oxygen content in the feedgas (upstream of the TWC) and tailpipe (downstream of the

Giovanni Fiengo is with School of Engineering, Università degli Studi del Sannio, Benevento, Italy. E-mail: gifiengo@unisannio.it. Jessy W. Grizzle is with Electrical Engineering and Computer Science Department, University of Michigan, Ann Arbor, MI 48109-2122, USA. E-mail: grizzle@umich.edu

Jeffrey A. Cook is with Ford Research Laboratory, Dearborn, MI, USA. E-mail: jcook2@ford.com

Amey Y. Karnik is with the Mechanical Engineering Department, University of Michigan, Ann Arbor, MI 48109-2122, USA. E-mail: akarnik@umich.edu

${ }^{1}$ The presented controller is a simplification of [7]. 
TWC) exhaust gas, respectively. In the case of a V-engine, the above description applies to each independent bank. In the case that two TWCs are present on an individual bank, a close-coupled catalyst and an under body catalyst for example, the downstream sensor may be placed between the two TWCs or downstream of both TWCs. This section collects the submodels used to construct an overall phenomenological model of the internal combustion engine (ICE), aftertreatment system, and sensor set. The simplified engine model focuses exclusively on $A / F$; it captures the four-cycle process delay and uses a lumped representation of the uncertainty due to injector characteristics and cylinder air charge estimation. The TWC model is taken from [2], and focuses on the oxygen storage phenomenon. The main peculiarity of the linear air-fuel ratio sensor model is the inclusion of a bias on the feedgas measurement [7].

\section{A. Internal Combustion Engine}

The ICE $A / F$ subsystem is modelled as a speed dependent induction-to-exhaust stroke delay of the fourstroke cycle cascaded with delays in the exhaust system. These delays account for the transport delays from the exhaust valve to the upstream EGO sensor, from the EGO sensor to the catalyst, within the catalyst body and from the TWC to the downstream sensor. For the particular system modelled here, the transport delay to the first sensor is lumped with the speed-dependent engine delay, the catalyst delay is lumped with the transport delay after the first EGO, and the delay from the TWC to the downstream EGO is considered negligible. For a four-cylinder engine sampled at an event interval (induction - compression - expansion exhaust) of 180 crankshaft degrees, the sample time, $d t$, is $\frac{30}{N}$ seconds $^{2}$, where $N$ is engine speed in rpm. Consequently, the engine delay is $4 d t$, or $\frac{120}{N}$ seconds, the lumped delay to the upstream sensor (engine plus transport) is $6 d t$ or, $\frac{180}{N}$ seconds. The lumped catalyst and transport delay after the sensor is $4 d t$.

A typical phenomenological engine model would include a representation of the intake manifold filling dynamics, in addition to the induction-to-exhaust stroke delay. The representation of intake manifold filling would be used to compute cylinder mass air charge in the model, and estimated cylinder mass air charge in the controller. In the controller, estimated cylinder mass air charge would be used in turn in a feedforward manner to form a base fuel charge command, which would then be corrected by a feedback controller on the basis of measured exhaust gas $A / F$; see [5]. In the controller implementation pursued here, the existing on-vehicle air-charge estimation scheme is used [10] without change, and only the feedback loops are modified. Hence, in the ICE model, only the uncertainty in the mass air charge estimate and the injector fuel flow need to be captured. These are represented by a lumped, additive, unmeasured perturbation to the commanded air fuel ratio, as shown in Figures 2 and 3.

\section{B. Three Way Catalytic Converter}

The catalytic converter model used in the design of the control strategy is taken from [2]. The model consists of two parts: an oxygen storage mechanism to account for the modification of the feedgas $A / F$ as it passes through the catalyst and the standard steady-state efficiency curves driven by the tailpipe $A / F$ computed from the oxygen storage model. The values of the steady-state efficiency curves have been updated from [2], based upon more recently measured data.

Oxygen storage sub-model: Let $0 \leq \Theta \leq 1$ be the fraction of oxygen storage sites occupied in the catalyst. The oxygen storage capacity is modelled as a limited integrator:

$$
\dot{\Theta}=\left\{\begin{array}{lr}
\frac{1}{C(M A F)} \rho\left(\lambda_{F G}, \Theta\right) \cdot 0.23 \cdot M A F \cdot\left(1-\frac{1}{\lambda_{F G}}\right) & 0 \leq \Theta \leq 1 \\
0 & \text { otherwise }
\end{array}\right.
$$

where $\dot{\Theta}$ represents $\frac{d \Theta}{d t} ; M A F$ denotes the mass air flow rate, used to approximate the flow rate of the mixture entering the TWC; $C$ represents the effective catalyst "capacity," or the volume of active sites for oxygen storage, expressed in terms of the mass of oxygen that can be stored in the catalyst, as a function of $M A F ; \rho$ describes the exchange of oxygen between the exhaust gas and the and $\lambda$ denotes the relative air-fuel ratio, with stoichiometry at $\lambda=1$ (the subscript $F G$ refers to the feedgas).

The effective TWC volume parameter, $C$, is expressed as a function of $M A F$ in order to account for an observed increase in effective volume at high flow rates, specifically above $10 \mathrm{~g} / \mathrm{s}$. This can be handled

\footnotetext{
${ }^{2}$ Note that at $1500 \mathrm{rpm}, \mathrm{dt}=20$ milliseconds.
} 
mathematically by combining this effect with the $M A F$ term by applying a saturation function to the $M A F$ input value. A function $f_{\text {sat }}(M A F)$ is created that is exactly equal to $M A F$ below $10 \mathrm{~g} / \mathrm{s}$ but maintains the value of $10 \mathrm{~g} / \mathrm{s}$ when $M A F>10 \mathrm{~g} / \mathrm{s}$. This function is shown in Table I. For clarity, it is emphasized that $C$ does not represent the physical volume of the catalyst, which is on the order of 2.5 liters. For example, if there were no usable storage sites (i.e. if they were poisoned by substances such as sulfur or phosphorus), then $C$ would be zero.

TABLE I

$f_{\text {sat }}(M A F)$

\begin{tabular}{|c|c|}
\hline$M A F(\mathrm{~g} / \mathrm{s})$ & $f_{\text {sat }}(M A F)(\mathrm{g} / \mathrm{s})$ \\
\hline 6.3 & 6.3 \\
\hline 10 & 10 \\
\hline 30.2 & 10 \\
\hline
\end{tabular}

The function $\rho$ is modelled as

$$
\rho\left(\lambda_{F G}, \Theta\right)=\left\{\begin{array}{ll}
\alpha_{L} f_{L}(\Theta) & \lambda_{F G}>1 \\
\alpha_{R} f_{R}(\Theta) & \lambda_{F G}<1
\end{array},\right.
$$

with $0 \leq f_{L} \leq 1$ representing the fraction of oxygen (combined or free) from the feedgas sticking to a site in the catalyst, and $0 \leq f_{R} \leq 1$ representing the fraction of oxygen being released from the catalyst and recombining with the feedgas. In $(2), f_{L}$ and $f_{R}$ vary with the fraction of occupied oxygen sites and potentially with the space velocity as well (that is, the feedgas volumetric flow rate divided by the catalyst volume). In the model, $f_{L}$ is assumed to be monotonically decreasing, with value one at $\Theta=0$ and zero at $\Theta=1$, and $f_{R}$ is assumed to be monotonically increasing, with value zero at $\Theta=0$ and one at $\Theta=1$. Typical functions $f_{L}$ and $f_{R}$, based upon data from [2], are shown in Figure 4. The specific equations used for $f_{L}$ and $f_{R}$, are as follows:

$$
\begin{gathered}
f_{L}(\Theta)=\frac{1-e^{6 \Theta}}{e^{6}-1}+1, \\
f_{R}(\Theta)=\frac{e^{-9 \Theta}-1}{e^{-9}-1} .
\end{gathered}
$$

The parameters $\alpha_{L}$ and $\alpha_{R}$ are included to represent the fact that the catalyst's storage and release rates of oxygen are different, with the release rate normally being higher than the storage rate.

The quantity $0.23 \times M A F \times\left(1-\frac{1}{\lambda_{F G}}\right)$, which can be rearranged to $0.23 \times M A F \times \frac{\Delta \lambda_{F G}}{\lambda_{F G}}$, represents the differential total mass of oxygen (combined or free) in the feedgas with respect to stoichiometry. When multiplied by $\rho$, it gives the mass of oxygen that is deposited in (or released from) the catalyst. By conservation of mass, the resulting equivalent tailpipe $A / F$ can be directly computed by the following:

$$
\lambda_{T P}=\lambda_{F G}-\rho\left(\lambda_{F G}, \Theta\right) \times\left(\lambda_{F G}-1\right) .
$$

Static conversion curves: For a typical three-way catalyst, there is a narrow window of air-fuel ratio around the stoichiometric point within which high conversion efficiencies of all three pollutants can be achieved. The static behavior of a TWC is characterized by the conversion efficiencies of $H C, C O$, and $N O x$ as functions of the tailpipe air-fuel ratio. These functions for the specific TWC used on the $5.4 L$ engine of Section VI are plotted in Figure 5 for a constant brick temperature. The maximum conversion efficiencies are $H C=99.56 \%$, $N O x=99.92 \%$ and $C O=98.98 \%$.

\section{Air-Fuel ratio sensor}

As shown in Figure 1, the engine is equipped with UEGO sensors to measure the quantity of oxygen in the exhaust gas at the feedgas and at the tailpipe. The linear sensor is modeled as a first order lag with unit gain and time constant $\tau$ equal to $250 \mathrm{~ms}$, as represented in the transfer function

$$
\lambda^{\text {meas }}=\frac{1}{\tau s+1} \lambda
$$


TABLE II

Measured bias on the feedgas UEGO SEnsor signal, as function of air-Fuel Ratio. Engine speed and LOAD ARE CONSTANT. RESUlts ARE FOR the 5.4L ENGine.

\begin{tabular}{c||c|c|c|c|c|c|c|c|c|c}
$A / F$ & 0.9449 & 0.9716 & 0.9853 & 0.9932 & 0.9974 & 0.9980 & 1.0000 & 1.0029 & 1.0049 & 1.0073 \\
Bias & -0.0122 & -0.0075 & -0.0065 & -0.0061 & -0.0058 & -0.0057 & -0.0057 & -0.0054 & -0.0051 & -0.0049 \\
\hline \hline$A / F$ & 1.0080 & 1.0129 & 1.0108 & 1.0114 & 1.0142 & 1.0184 & 1.0349 & 1.0689 & 1.0897 & 1.1046 \\
Bias & -0.0047 & -0.0043 & -0.0041 & -0.0038 & -0.0037 & -0.0031 & -0.0015 & -0.0007 & -0.0018 & -0.0018
\end{tabular}

TABLE III

Measured bias on the feedgas UEGO sensor signal, as function of engine speed and load. Air-Fuel RAtio is Close to Stoichiometry. Results ARE FOR THE 5.4L ENGine.

\begin{tabular}{c|cc} 
Engine Speed & Load & Bias \\
\hline \hline \multirow{2}{*}{750} & 0.2 & -0.004 \\
& 0.45 & -0.0065 \\
\hline \hline \multirow{3}{*}{1500} & 0.2 & -0.0065 \\
& 0.45 & -0.0047 \\
& 0.7 & -0.0045 \\
\hline \hline \multirow{3}{*}{2250} & 0.2 & -0.002 \\
& 0.45 & -0.0038 \\
& 0.7 & -0.0035
\end{tabular}

where $\lambda^{\text {meas }}$ is the measured variable and $\lambda$ is the actual $A / F$ in the feedgas or in the tailpipe. Importantly, feedgas and tailpipe $A / F$ measurements are affected by different types of inaccuracy. Upstream of the catalyst, non-equilibrium effects in the exhaust gas result in a systematic bias error in the sensor [4], [8], [9], [11], [14]. This bias is in part due to incomplete catalysis of $C O$ on the sensor substrate and in lesser part to $N O x$; an additional confounding factor is the large discrepancy in the diffusion rate of $\mathrm{H}_{2}$ with respect to other species present in the exhaust gas. Moreover, the bias varies as a function of air-fuel ratio, as reported in Table II and shown in Figures 6 and 7, and engine speed and load, as reported in Table III. It is hypothesized that the bias also varies with ageing of the sensors and the TWC, and perhaps with fuel formulation. The variation in the bias with operating condition makes online estimation of the bias essential.

Assuming thermodynamic equilibrium in the exhaust gas after the catalyst, the measurement disturbance at the tailpipe $A / F$ sensor is considered to be zero mean white noise, in particular, the bias phenomenon associated with the feedgas is negligible at the tailpipe. Hence, the model of the UEGO sensor is the following:

$$
\begin{aligned}
\lambda_{F G}^{\text {meas }} & =\frac{1}{\tau s+1} \lambda_{F G}+b+w_{F G} \\
\lambda_{T P}^{\text {meas }} & =\frac{1}{\tau s+1} \lambda_{T P}+w_{T P},
\end{aligned}
$$

where $w$ represents zero mean white noise on the feedgas and tailpipe sensor measurement and $b$ is the systematic bias on the feedgas measurement.

\section{Control Strategy for a Single Catalyst}

In this section, a controller is designed with two objectives in mind: first, to simultaneously maximize the conversion efficiencies of $H C, C O$ and $N O x$; and second, to obtain steady-state air-fuel control that is robust with respect to disturbances, while minimizing excursions around stoichiometry. This latter objective is, essentially, a driveability issue: when the engine is operating in steady-state (constant mass air flow), large $A / F$ oscillations cause torque and, hence, rpm variations.

A series controller topology is adopted ${ }^{3}$; see Figure 8. The objective of the first block, the Fore Controller, is to respond relatively quickly to $A / F$ disturbances on the basis of measured feedgas oxygen level. As discussed

\footnotetext{
${ }^{3}$ The standard feedforward control action based on measured or estimated mass air flow rate is not discussed; see [5], [10]. A
} 
in the previous section, feedgas oxygen measurement is corrupted by a systematic bias. The objective of the second block, the Aft Controller, is to adjust the setpoint of the fore-controller, on the basis of both $A / F$ measurements, so that the TWC achieves simultaneously high conversion efficiencies for $H C$ and $N O x$. The aft controller acts on a slower time scale commensurate with the longer measurement delay in the second sensor.

Fore Controller: The fore controller is realized by a switching-type PI controller. Measured feedgas $A / F$ ratio, corrected for the bias via $\lambda_{F G}^{\text {meas }}(t)-\hat{b}(t)$, where $\hat{b}(t)$ is an estimate of the bias $b(t)$, is first compared to a reference value provided by the aft controller $\lambda_{\text {ref }}^{\text {fore }}(t)$, resulting in an error signal of -1 if measured $A / F$ is lean of the reference and +1 otherwise. The error signal is fed into a standard PI controller,

$$
\begin{aligned}
\lambda_{c}(t) & =\left(K_{P}(\text { rpm })+\frac{K_{I}(\text { rpm })}{s}\right) \cdot \operatorname{sign}(\operatorname{error}(t))+\lambda_{\text {ref }} \\
\operatorname{error}(t) & =\lambda_{\text {ref }}^{\text {fore }}(t)-\left(\lambda_{F G}^{\text {meas }}(t)-\hat{b}(t)\right)
\end{aligned}
$$

with gains, $K_{P}$ and $K_{I}$, scheduled as a function of engine speed, so as to create a limit cycle with a (sensed) peak-to-peak amplitude of approximately $0.2 \mathrm{~A} / \mathrm{F}$ and period 1.5 seconds. $\lambda_{c}(t)$ is the commanded $A / F$ ratio (multiplier to the feedforward signal) and $\lambda_{\text {ref }}$ is the reference signal, typically equal to stoichiometry, or just slightly rich of stoichiometry (less than one part in a thousand). The output of the controller is limited, and hence anti-windup is implemented.

Aft Controller: The aft controller is composed of a bias estimator and a proportional term. The bias estimator uses upstream and downstream $A / F$ measurements to correct the measurement of the upstream oxygen sensor. The proportional controller feeds back the post-catalyst UEGO sensor measurement and establishes the reference for the fore controller.

The bias estimator is based on the following observations. In the absence of a bias, the averages of the $A / F$ in the feedgas and tailpipe should be the same whenever the feedgas signal remains constant for a period of time sufficiently long to fill an empty catalyst with oxygen or, conversely, to deplete a filled one. That is, if $\lambda_{F G}$ is constant at a lean value, $\lambda_{T P}$ will reach the same value when the catalyst is completely filled with oxygen. Conversely, if $\lambda_{F G}$ is rich, $\lambda_{T P}$ and $\lambda_{F G}$ will be equal after the catalyst has been entirely depleted of oxygen. At stoichiometry, $\lambda_{T P}$ equals $\lambda_{F G}$, independently of the oxygen state of the catalyst. Hence, in steady-state, a difference in the averaged values of the two measurements is due to the bias in the upstream sensor.

During transients, the bias estimator acts to indirectly control the level of oxygen stored in the catalyst. For example, suppose that $\lambda_{F G}$ oscillates symmetrically around stoichiometry and that the catalyst is (nearly) depleted of oxygen. Then, the average of $\lambda_{T P}$ will be slightly rich due to the fact that the TWC can only absorb oxygen when the feedgas is lean of stoichiometry. Consequently, the bias estimate will be larger than the actual bias, which has the same effect as increasing the setpoint of the fore controller to a leaner value; it follows that the $A / F$ system is driven toward filling the catalyst with oxygen. Similar reasoning applies when the catalyst is (nearly) saturated with oxygen. Hence, as opposed to updating the bias estimate only in steady state as reported in [7], here, the bias is updated continually.

The estimated bias is thus computed as follows

$$
\hat{b}(t)=\overline{\lambda_{F G}^{\text {meas }}}(t-\delta)-\overline{\lambda_{T P}^{\text {meas }}}(t)
$$

where $\hat{b}$ is the estimated bias, $\delta$ is the catalyst transport delay and is approximated as $4 d t$ for the analysis. The $\overline{\lambda_{F G}^{m e a s}}$ and $\overline{\lambda_{T P}^{\text {meas }}}$ are respectively the average of the measurements of the pre- and post- catalyst sensors, approximated by low-pass filtering. The estimated bias is limited to a maximum and minimum range, based on measurements such as those reported in Tables II and III. The bias estimate is subtracted from the output of the upstream UEGO sensor before it is used by the fore controller.

The proportional term is formed by subtracting the measured tailpipe $A / F$ from the reference value and then multiplying by an "asymmetric" gain; that is, lean errors are corrected more aggressively than rich errors

parallel or inner-outer controller topology was analyzed in an internal report; the series configuration was found to give better results for this application. 
to avoid NOx breakthrough. The proportional term is then added to the reference value for tailpipe $A / F$, and provided to the fore-controller:

$$
\lambda_{\text {ref }}^{\text {fore }}=K_{\text {aft }}\left(\lambda_{\text {ref }}^{\text {aft }}-\lambda_{T P}^{\text {meas }}\right)+\lambda_{\text {ref }}^{\text {aft }}
$$

where $\lambda_{\text {ref }}^{\text {fore }}$ is the reference value for the fore-controller determined by the proportional controller; $K_{a f t}$ is the proportional gain; $\lambda_{\text {ref }}^{\text {aft }}$ is the reference value for the aft-controller, typically equal to stoichiometry.

\section{Simulation of the Control Strategy}

This section uses simulation to tune the parameters in the controller and to investigate the performance of the closed-loop system. For the catalyst model, recall that the steady-state conversion efficiencies at stoichiometry are $H C=99.56 \%, N O x=99.92 \%$ and $C O=98.98 \%$. The goal is to achieve these values in the face of significant measurement uncertainty in the upstream UEGO sensor and significant error in the estimated air-charge and/or fuel injection quantity. The combined effects of uncertainty in the injected fuel quantity and estimated air-charge have been simulated by summing the signal shown in Figure 3 with the output of the PI controller. A bias equal to $-0.005 \lambda$ is assumed for the upstream UEGO. White noise disturbances taking values between $\pm 0.5 \%$ of stoichiometry are applied to the UEGO sensors. The simulations used mass air flow and engine speed corresponding to Bags 2 and 3 of an FTP cycle for a $5.4 L$ engine (see Figure 20 in Section VI). The controller gains were tuned, by use of a genetic algorithm, to maximize summed $N O x$ and $H C$ conversion efficiency, subject to an upper bound on the variance of the feedgas $A / F$. As this is standard, it is not reported here.

Simulation results are shown in Figures 9 - 11. The controller shows excellent performance. Even in the presence of very significant uncertainty, the tailpipe $A / F$ is regulated very closely to stoichiometry, and very nearly achieves the maximal possible average simultaneous conversion efficiencies of the three main pollutants, that is $99.36 \%$ for unburned hydrocarbons and $99.80 \%$ for nitrogen oxide. Moreover, the variance of the feedgas $A / F$ is small (see Figure 9), indicating that there should be no driveability problems due to the actions of the controller. The Figure 11 shows simulated oxygen storage level and the estimated bias level.

The results of a robustness analysis are now reported. For this purpose, while holding all values in the controller fixed, the bias, measurement noise and injector uncertainty disturbances were increased, and the oxygen storage capacity of the catalyst and the engine time delays were modified. To further evaluate robustness to model uncertainty, a completely different TWC representation (described in [6]) from that used when tuning the controller was inserted in the simulation model and the position of the second $A / F$ sensor was changed by inserting a delay equal to $4 d t$ sec between the TWC and this sensor. For conciseness of presentation, the results of the simulations are tabulated with respect to the conversion efficiencies for $N O_{x}$, and $H C$, and the mean and standard deviations of the tailpipe and feedgas air-fuel ratios, see Table IV. For a baseline, the first column of the table contains the performance metrics for the controller subjected to the standard disturbances and uncertainties of: bias equal to $-0.005 \lambda$; white noise sensor disturbance between $\pm 0.5 \%$ of stoichiometry, and injector uncertainty as illustrated in Figure 3. The time delays and the oxygen storage capacity of the catalyst are fixed to values of Section II and [2], respectively.

In the robustness investigation, the various disturbances are first applied separately. The second column of Table IV shows the simulation results for bias equal to $-0.007 \lambda$, and white noise sensor disturbance and injector uncertainty increased by $100 \%$. The results in the third column are obtained using the standard disturbances enumerated above, but decreasing by $50 \%$ the oxygen storage capacity, thereby simulating the aging of the catalyst. The performance of the system when the engine delays are increased by $50 \%$ and the sensor placement is modified, is illustrated in the fourth column of Table IV. The fifth column reports results when the modified catalyst dynamics are substituted. Finally, the last column provides the results when all of the changes are applied simultaneously.

\section{Control Strategy FOR two CATAlysts in SERIES}

Consider now the system formed by the cascade of the engine and two catalytic converters, as shown in Figure 12. In practice, the first catalyst is typically smaller and is placed close to the engine for early light-off (close coupled catalyst, CC-TWC) and the second is placed further down stream for best operation under fully warmed-up conditions (under body catalyst, UB-TWC). Hence, two different transport delays are needed in 
TABLE IV

Robustness anALYSis COMPARING THE BASE-LINE RESUlts TO SUCCESSIVE CHANGES IN THE DisturbanCE AND NOISE LEVELS, OXYGEN STORAGE CAPACITY OF THE CATALYST, TIME DELAYS IN THE MODEL, CHOICE OF CATALYST OXYGEN STORAGE MODEL, AND, FINALLY, IMPOSING ALL OF THE UNCERTAINTIES AT ONCE.

\begin{tabular}{l||c|c|c|c|c|c}
\multicolumn{1}{c||}{ Performance } & \multicolumn{7}{c}{ Simulations to Evaluate Robustness } \\
\cline { 2 - 7 } & Base-Line & Disturb & TWC Cap. & Delays & TWC Model & All Together \\
\hline \hline$H C$ Eff. & 99.3314 & 99.3622 & 99.3987 & 99.2597 & 99.5005 & 99.4165 \\
$N O_{x}$ Eff. & 99.7966 & 99.3127 & 99.7572 & 99.8344 & 99.9020 & 99.2765 \\
\hline$\lambda_{F G}$ mean & 1.0009 & 1.0012 & 1.0009 & 1.0009 & 1.0003 & 1.0005 \\
$\lambda_{F G}$ std. dev. & 0.0127 & 0.0147 & 0.0127 & 0.0131 & 0.0128 & 0.0166 \\
$\lambda_{T P}$ mean & 0.9999 & 0.9999 & 0.9999 & 0.9998 & 1.0000 & 1.0000 \\
$\lambda_{T P}$ std. dev. & 0.0003 & 0.0004 & 0.0002 & 0.0003 & 0.0000 & 0.0003
\end{tabular}

the model. In particular, there is a small transport delay between the engine and the first TWC and another delay between the two catalysts.

The objective is to realize a control strategy for this dual-catalyst system that maximizes conversion efficiencies while maintaining a minimal $A / F$ limit cycle amplitude. To compute the overall conversion efficiencies, let the mass of a generic chemical species be $m_{X}^{f g}, m_{X}^{i n t}$ and $m_{X}^{t p}$, respectively, at the feedgas, at the output of the CC-TWC and at the tailpipe. The overall conversion efficiency, $\eta$, is computed as follows

$$
\begin{aligned}
\eta & =\left(\frac{m_{X}^{f g}-m_{X}^{t p}}{m_{X}^{f g}}\right) \times 100 \% \\
& =\left(1-\frac{m_{X}^{t p}}{m_{X}^{f g}}\right) \times 100 \%
\end{aligned}
$$

Now, letting the conversion efficiency of the first and the second TWC be, respectively, $\eta_{C C}$ and $\eta_{U B}$, the mass of the pollutants at the output of the TWCs can be calculated as

$$
\begin{aligned}
m_{X}^{i n t} & =m_{X}^{f g}\left(1-\frac{\eta_{C C}}{100}\right) \\
m_{X}^{t p} & =m_{X}^{i n t}\left(1-\frac{\eta_{U B}}{100}\right) \\
& =m_{X}^{f g}\left(1-\frac{\eta_{C C}}{100}\right)\left(1-\frac{\eta_{U B}}{100}\right) .
\end{aligned}
$$

Finally, substituting the equation (13) in (12), the overall conversion efficiency is obtained as

$$
\begin{aligned}
\eta & =\left(1-\frac{m_{X}^{f g}\left(1-\frac{\eta_{C C}}{100}\right)\left(1-\frac{\eta_{U B}}{100}\right)}{m_{X}^{f g}}\right) \times 100 \% \\
& =\left(\frac{\eta_{C C}}{100}+\frac{\eta_{U B}}{100}-\frac{\eta_{C C}}{100} \frac{\eta_{U B}}{100}\right) \times 100 \%
\end{aligned}
$$

\section{A. Controlling only the $C C-T W C$}

In the following, the fore-aft controller of Section III is applied to the feedgas UEGO and the UEGO at the output of the CC-TWC. For comparison with the previous results for a single catalyst, it will be assumed that the total oxygen storage capacity of the two catalysts is equal to that of the single TWC studied in Section IV. In particular, it is assumed that the capacity of the CC-TWC is one-third of the total, while the remaining two-thirds is the capacity of the UB-TWC. In addition, it will be assumed that the close-coupled and underbody catalysts have equal conversion efficiencies and that the cascade of the two catalysts has the same overall efficiency as the single TWC for each of $N O x$ and $H C$. In particular, in (14), for each pollutant, $\eta_{C C}=\eta_{U B}$ (conversion efficiencies at stoichiometry are $H C=93.37 \%$ and $N O x=97.12 \%$ ), with $\eta$ as in Figure 5 . 


\section{TABLE V}

ROBUSTNESS ANALYSIS OF THE CASCADE OF TWO TWCS WHEN CONTROLLED ON THE BASIS OF A UEGO IN THE FEedgas and a SECONd UEGO Between the two CATAlysts. The table COMPARES the Base-Line RESUlts OF THE CONTROL STRATEGY TO SUCCESSIVE CHANGES IN THE DISTURBANCE AND NOISE LEVELS, OXYGEN STORAGE CAPACITY OF THE CATALYST, TIME DELAYS IN THE MODEL, CHOICE OF CATALYST OXYGEN STORAGE MODEL, AND, FINALLY, IMPOSING ALL OF THE UNCERTAINTIES AT ONCE.

\begin{tabular}{l||c|c|c|c|c|c}
\multicolumn{1}{c||}{ Performance } & \multicolumn{7}{c}{ Simulations to Evaluate Robustness } \\
\cline { 2 - 7 } & Base-Line & Disturb & TWC Cap. & Delays & TWC Model & All Together \\
\hline \hline$H C$ Eff. & 99.5505 & 99.5450 & 99.5526 & 99.5501 & 99.5580 & 99.5556 \\
$N O_{x}$ Eff. & 99.9120 & 99.8925 & 99.9095 & 99.9120 & 99.9132 & 99.9013 \\
\hline$\lambda_{F G}$ mean & 1.0008 & 1.0009 & 1.0008 & 1.0009 & 1.0002 & 1.0004 \\
$\lambda_{F G}$ std. dev. & 0.0127 & 0.0147 & 0.0126 & 0.0131 & 0.0125 & 0.0154 \\
$\lambda_{T P}$ mean & 1.0000 & 1.0000 & 1.0000 & 1.0000 & 1.0000 & 1.0000 \\
$\lambda_{T P}$ std. dev. & 0.0000 & 0.0000 & 0.0000 & 0.0000 & 0.0000 & 0.0000
\end{tabular}

The parameters of the controllers were re-tuned for the new model using a genetic algorithm. The simulations use the disturbances of Section IV: injector uncertainty as shown in Figure 3; bias equal to $-0.005 \lambda$; a white noise disturbance, taking values between $\pm .5 \%$ of stoichiometry is applied to the UEGOs. Figures 13 - 15 present the simulation results: the air-fuel ratio; the conversion efficiencies; the relative oxygen levels and the estimated bias. The conversion efficiencies reached in this configuration are very close to the maximal possible average simultaneous conversion efficiencies, that is $99.55 \%$ for unburned hydrocarbons and 99.91 for nitrogen oxide. As in Section IV, a robustness analysis was performed; the results are presented in Table V.

Another way to control the cascade on the basis of only two sensors is to place a UEGO in the feedgas and a second UEGO after the UB-TWC. Due to the choice of model parameters, controlling the cascade of the two TWCs on the basis of these two measurements gives very nearly the same results as controlling the single TWC of Section IV, and hence the corresponding plots and tables are not reported. A comparison of the simulation results suggests a somewhat surprising conclusion that superior control of the cascade is obtained by placing the sensor between the two TWCs, so that only the CC-TWC is actively controlled. In fact, this is born out in the experiments presented in Section VII.

\section{B. Loss of Efficiency in the $C C$ - $T W C$}

Clearly, the above result depends on the relative sizes of the two catalysts (just consider replacing the CC-TWC by a straight pipe, for example). It is interesting to investigate how to best control the overall aftertreatment system if the close-coupled catalyst experiences a reduction in efficiency, either temporarily due to high temperatures that it may reach under certain operating conditions due to its position close to the engine, or permanently due to ageing or poisoning. To this aim, it is supposed that an indirect estimate of catalyst efficiency is available, based for example on temperature or estimated oxygen storage capacity [6], $[12],[15]$. When the efficiency of the CC-TWC is sufficiently impaired, it is proposed that the aft-controller should feed back the TP sensor instead of the mid-bed sensor (see Figure 16).

In order to illustrate the effect of such a control strategy, a simulation of the system is performed where efficiency of the CC-TWC changes as in Figure 17. In particular, for the first 200 seconds, the catalyst's efficiency is normal, and hence the aft-controller feeds back the mid-bed sensor. At time 200 seconds, the CC-TWC's efficiency decreases precipitously by $80 \%$, so that the overall maximum possible $H C$ efficiency becomes $94.6054 \%$ while for $N O x$ it becomes $97.6814 \%$. For purposes of illustration, from 200 to 600 seconds, the aft-controller continues to feedback the mid-bed sensor. At time 600 seconds, the problem is diagnosed and the aft-controller feeds back the TP air-fuel sensor measurement. Table VI summarizes the performance of the system operating over the three time regimes. It is seen that when the switch is made to controlling on the TP sensor, the achieved conversion efficiencies are again close to their maximum possible values. 
TABLE VI

Performance of the System Changing the EFficiency of the CC-TWC: THe First 200 Seconds the CATALYST'S EFFICIENCY IS NORMAL; FROM 200 TO 600 SECONDS, THE AFT CONTROLLER FEEDS BACK THE MID-BED SENSOR BUT THE CC-TWC'S EFFICIENCY IS DECREASED BY 80\%; AT TIME 600 SECONDS, THE CONTROLLER STARTS TO FEEDBACK THE TP SENSOR.

\begin{tabular}{l||c|c|c} 
Performance & $0 \leq t<200$ & $200 \leq t<600$ & $600 \leq t<1350$ \\
\hline \hline$H C$ Eff. & 99.5386 & 93.6335 & 94.5262 \\
$N O_{x}$ Eff. & 99.8950 & 97.2147 & 97.0292 \\
\hline$\lambda_{F G}$ mean & 1.0010 & 1.0001 & 1.0009 \\
$\lambda_{F G}$ std. dev. & 0.0133 & 0.0128 & 0.0125 \\
$\lambda_{T P}$ mean & 1.0000 & 0.9991 & 1.0000 \\
$\lambda_{T P}$ std. dev. & 0.0001 & 0.0004 & 0.0002
\end{tabular}

\section{Experimental Facility and Methodology}

Experiments were performed in two different dynamometer test cells. In the first set of experiments, a $5.4 L$ V8 engine and automatic transmission were connected to a motoring dynamometer, and in the second set of experiments conducted a year later, a $2.3 \mathrm{~L}$ in-line four cylinder engine and automatic transmission were connected to a motoring dynamometer. In each case, the dynamometer's controller allowed speed-torque profiles approximating standard test cycles to be run, specifically, the warmed-up portion of the Federal Test Procedure (FTP) cycle. The FTP is comprised of three modes, or "bags" (referring to the fluropolymer bags in which emissions are stored for analysis). Bag 1 comprises a cold engine start and 505 second drive attaining a speed of about $57 \mathrm{mph}$. Bag 2 is a low speed, hot stabilized, drive cycle lasting 867 seconds. For bag 3, the engine is stopped for 10 minutes, after which a hot start is performed and the 505 second bag 1 is repeated. The experimental results described here are measured over bags 2 and 3, without the ten minute pause between the two bags.

5.4L V8 engine: The right side of the engine was used for evaluating the dual-UEGO $A / F$ control strategy described in Section III, along with a few variants. The exhaust system was modified to consist of a single underbody TWC, mounted approximately $30 \mathrm{~cm}$ downstream of the exhaust manifold; see Figure 18. A close-coupled catalyst for rapid light off upon cold start was not used. The underbody TWC shown in Figure 18 consisted of four bricks of approximately equal volume but with different precious metal loadings. The oxygen storage capacities of bricks two and three were the same; brick one had $20 \%$ greater capacity and brick four had four times the capacity. The aftertreatment system had been aged for greater than 100 hours prior to testing. Thermocouples were inserted ahead of each brick into the exhaust stream, and in the catalyst substrate $2.5 \mathrm{~cm}$ from the face of each brick. An emissions tap and two sensor bosses were installed between bricks one and two (referred to hereafter as the brick one location) and between bricks two and three (referred to hereafter as the brick two location); this allowed UEGO and HEGO data to be recorded simultaneously. Feedgas emissions and $A / F$ were measured approximately $10 \mathrm{~cm}$ upstream of the catalyst. Emissions could be simultaneously recorded at two of the three points in the exhaust system, namely, feedgas, and one of brick one or brick two, for the purpose of determining conversion efficiencies of $H C$ and $N O x$ ( $C O$ was recorded but not analyzed).

The UEGO sensors were supplied with a nominal calibration curve. The calibration was refined in the test cell in the following manner. First of all, approximate linearity was verified through a standard $A / F$ sweep over the range from $12 A / F$ to $18 A / F$, where exhaust $A / F$ was estimated from measured air mass flow rate and fuel pulse width. Stoichiometry was then more precisely estimated from catalyst conversion characteristics, namely, by correlating UEGO-sensor voltage readings to the point where the catalyst exhibited best simultaneous conversion of $H C$ and $N O x$, as determined by the emissions benches. Finally, stoichiometry was also estimated through HEGO measurements. Using the HEGO sensor and a standard PI controller, an $A / F$ limit cycle was established about stoichiometry. The UEGO sensor output was then sampled synchronously with the HEGO crossing stoichiometry, and averaged over ten crossings in each direction. The resulting estimated stoichiometric point for the UEGO agreed within $0.03 \pm 0.002 \%$ of the value estimated from the catalyst's 
best conversion point.

The base software of the engine control unit was modified to allow the engine to be run with the standardbaseline - controller, or to accept a replacement feedback signal generated externally to the engine control unit. More precisely, in the latter case, the feedforward portion of the onboard $A / F$ control loop based on air charge estimation was retained, the onboard feedback loop was disabled, and the commanded fuel pulse width was multiplied by the value of the external signal. Hence when the external signal was one, the engine operated under nominal feedforward control, and by varying the external signal about one, commanded $A / F$ could be varied rich or lean of the feedforward value, just as in a standard negative feedback loop. The external signal was generated in a real-time control prototyping system via the algorithm of Section III.

The baseline controller implements a proprietary algorithm based on dual-HEGOs, one in the feedgas and one in the tailpipe. The algorithm was calibrated to function with the tailpipe HEGO at the brick two location.

2.3L Four Cylinder Engine: The exhaust aftertreatment system studied on this engine consisted of a close-coupled, two-brick catalyst mounted directly to a (log-style) exhaust manifold; see Figure 19. The catalyst had been aged approximately $150,000 \mathrm{~km}$. Emissions taps were available in the feedgas just before the catalyst, in the mid-bed just after brick one (referred to hereafter as the brick one location), and at the exit of the catalyst, following brick two (referred to hereafter as the brick two location). The aftertreatment system on this engine also includes a second catalyst mounted approximately $40 \mathrm{~cm}$ downstream of the first catalyst, which was not included as part of this study. UEGO sensors were mounted in the feedgas, brick one and brick two locations of the close-coupled catalyst; calibration of the sensors was performed as described previously. The standard on-board $A / F$ controller implemented essentially the same proprietary, dual-HEGO algorithm that was used on the $5.4 \mathrm{~L}$ engine. The only difference is that, a year after the tests run on the $5.4 \mathrm{~L}$ engine, the second HEGO sensor had been moved to the brick one location, and the controller's calibration adjusted accordingly.

In this test cell, it was not possible to make modifications to the base software. External control of the engine's $A / F$ via the algorithm of Section III could only be effected in an indirect manner. The error signal in (9) was used to create a synthetic feedgas HEGO signal, which was then applied to the corresponding input port of the onboard controller. Specifically, negative (rich) errors were mapped to 0.9 and positive (lean) errors were mapped to 0.3 ; recall that the output voltage of a standard HEGO varies between $0.1 \mathrm{~V}$ and $0.9 \mathrm{~V}$, with stoichiometry approximately $0.45 \mathrm{~V}$. In this way, the existing onboard controller, when set to operate on the basis of a single HEGO placed in the feedgas, would approximately implement the desired algorithm.

\section{Experimental Results}

The dual-UEGO control strategy of Section III was tested on the engines and exhaust systems described in Section VI over bags 2 and 3 of the FTP drive cycle. Figure 20 depicts measured engine speed over the cycle for the $5.4 L$ engine. The experiments on the $5.4 L$ engine were performed over a five week period in AugustSeptember 2002, concurrently with other work in the test cell, while the experiments on the four-cylinder $2.3 \mathrm{~L}$ engine were performed in July and August 2003. Cold start was not an objective of the testing plan, and hence multiple tests could be run in a single day (typically three to five, when a test cell was available). Since the catalyst would be in a "random" thermal state depending on the length of time since the previous test, the first 100 seconds of the cycle are neglected in the presentation of the results.

5.4L V8 engine: On the $5.4 L$ engine, the controller was operated with several variations: the tailpipe UEGO placed in brick one or brick two; bias estimation enabled always or only in steady state; and the aft controller with and without the proportional term. The baseline controller was always operated with the tailpipe $A / F$ measurement at brick two. The setpoint of the dual-UEGO controller was selected to be stoichiometry, that is, $\lambda=1$. Recall that the bias varies as a function of speed, load and air-fuel ratio as presented in Tables II and III. The initial experimental results are presented in Tables VII through IX. The reported conversion percentage is the average of the instantaneous conversion percentages; the overall conversion percentage can be computed from the given data. The best emissions results were obtained with the full algorithm, that is, with a proportional term in the aft controller and with bias estimation enabled even during transients. The resulting emissions were consistently lower than those achieved by the baseline controller. The full algorithm achieved from 10 to 33 times less cumulative tailpipe $N O x$ than the baseline controller, and from 1.15 to 6.2 times less cumulative tailpipe $H C$ than the baseline controller. 
Figures 21 to 25 provide more details for the second experiment of Table VII, comparing the dual-UEGO controller of Section III to the baseline controller. Figure 26 depicts a typical bias estimate throughout a portion of the FTP cycle. Based on the simulation study of Section IV, it is conjectured that the oscillations in the bias estimate are correlated with oscillations in the level of stored oxygen in the catalyst, which is known to promote sustained catalytic activity; see [3]. The emissions results for other configurations of the controller are reported in order to confirm the "best" form of the controller and to demonstrate that the algorithm is robust in that similar results were obtained with various configurations. The two most important aspects of the reported control algorithm appear to be the ability to make small adjustments to the set point of the fore controller on the basis of the tailpipe $A / F$ sensor, which is greatly facilitated by the use of a linear sensor in the feedgas, and the placement of the second sensor after brick one of the catalyst. Recall that this latter configuration was suggested by the simulation results of Section V.

2.3L Four cylinder engine: The controller with full algorithm was implemented on the four-cylinder $2.3 \mathrm{~L}$ engine. Similar to the tests conducted on the $5.4 \mathrm{~L}$ engine, the controller was run with tailpipe UEGO placed in brick one or in brick two. Other variations included using UEGO sensor combinations of different manufacturers to validate the compatibility of different sensors to the controller. The use of a switching HEGO placed in the tailpipe in addition to the emission benches reduced the iterations encountered in identifying the setpoint for the sensors. The experimental results are reported in Tables X and XI. The only reading for the baseline controller shows that it operates rich of stoichiometry in order to robustly reduce $N O x$ emissions. The control algorithm, with tailpipe $A / F$ measured at brick one, achieved from 9.6 times more to 1.4 times less cumulative NOx than the baseline controller, and from 2.7 to 10.9 times less cumulative $H C$ than the baseline controller. The cumulative tailpipe emissions (averaged over the set of similar experiments) are shown in Figures 27 and 28.

\section{Conclusions}

The static conversion efficiency of modern three way catalytic convertors can be extremely high, on the order of $99.9 \%$ and higher. The conversion efficiency is very sensitive to persistent variations in air-fuel ratio from stoichiometry on the order of one part in a thousand, or less. This places enormously stringent requirements on the air-fuel ratio control system. An important obstacle to achieving such precise control of air-fuel ratio is the rich bias that occurs on the feedgas oxygen sensor, which sends the closed-loop system lean. The magnitude of the bias varies with many factors, and typically ranges from one to ten parts per thousand.

A novel method of actively regulating catalyst performance on the basis of measured $A / F$ has been reported. The use of dual UEGO sensors, one in the feedgas and one downstream of (at least part of) the catalyst has permitted the bias, that is present in the measurement of feedgas $A / F$, to be estimated on-line and fed forward to a standard switching-type PI-controller, acting on the corrected feedgas $A / F$ measurement. Extensive simulations showed that the proposed controller exhibits excellent nominal performance and considerable robustness to changes in the parameters of the system. The simulation analysis also suggested a somewhat surprising placement for the second UEGO sensor in an aftertreatment system consisting of a cascade of multiple catalysts: the sensor should be placed between two of the catalysts and not downstream of the last catalyst. In practice, modern catalysts consist of several bricks, and thus the result suggests placing the second sensor in the middle of a single catalyst.

The proposed controller has been evaluated in two dynamometer test cells, one equipped with a $5.4 L$ V8 engine and the other with a $2.3 \mathrm{~L}$ in-line four-cylinder engine. In each case, the performance of the controller has been evaluated against a base-line controller that uses dual-HEGOs. A marked performance improvement in $H C$ and $N O x$ conversion efficiency has been demonstrated with the dual UEGO controller for the $5.4 L$ V8 engine, with the best performance achieved with the second UEGO placed in the mid-bed of a catalyst instead of downstream of the catalyst, thereby confirming the prediction of the simulation analysis. The baseline controller on the $2.3 \mathrm{~L}$ engine was calibrated slightly rich of stoichiometry, thereby providing robustly high $N O x$ efficiency, at the expense of $H C$ conversion and fuel economy. Dual-UEGO control at stoichiometry improved $H C$ conversion efficiency compared to the baseline, at the cost of $N O x$, as would be expected.

\section{ACKNOWLEDGMENTS}

The authors sincerely thank Phil Husak, Don Lewis, Dan Meyer, Dennis Reed, Robert Wiley and Roy Sowersby for their very valuable assistance, in the test cells and in numerous other ways, that led to the 
successful completion of this project. Deepak Aswani is thanked for his assistance in programming the initial real-time control computations. G. Fiengo, Amey Karnik and J.W. Grizzle gratefully acknowledge funding under a Ford Motor Company URP titled Active Control of Aftertreatment for Improved Emissions and Fuel Economy.

\section{REFERENCES}

[1] M. Ammann, H.P. Geering, C.H. Onder, C.A. Roduner, and E. Shafai. Adaptive control of a three-way catalytic convertor. American Control Conference, 2000.

[2] E.P. Brandt, Y. Wang, and J.W. Grizzle. Dynamic modeling of a three-way catalyst for si engine exhaust emission control. IEEE Trans. on Control System Technology, 8(5):767-776, 2000.

[3] B. Campbell, R. Farrington, G. Inman, S. Dinsdale, D. Gregory, D. Eade, and J. Kisenyi. Improved three-way catalyst performance using an active bias control regeneration system. SAE paper 2000-01-0499, 2000.

[4] A.D. Colvin, J.W. Butler, and J.E. Anderson. Catalytic effects on ZrO2 oxygen sensors exposed to non-equilibrium gas mixtures. J. Electroanal. Chem., (136), 1982.

[5] J.A. Cook, J.W. Grizzle, and J. Sun. Automotive engine control. The Control Handbook, CRC Press, W. Levine, pages 1261-1274, 1996.

[6] G. Fiengo, L. Glielmo, and S. Santini. On board diagnosis for three-way catalytic converters. International Journal of Robust Nonlinear Control, 11:1073-1094, 2001.

[7] G. Fiengo, J.W. Grizzle, and J.A. Cook. Fore-aft oxygen storage control. American Control Conference, 2002.

[8] H. Germann, S. Tagliaferri, and H.P. Geering. Differences in pre- and post-converter lambda sensor characteristics. SAE paper 960335, 1996.

[9] H.J. Germann, C.H. Onder, and H.P. Geering. Fast gas concentration measurements for model validation of catalytic converters. SAE paper 950477, 1995.

[10] J.W. Grizzle, J.A. Cook, and W.P. Milam. Improved cylinder air charge estimation for transient air fuel ratio control. American Control Conference, 1994.

[11] D.R. Hamburg, J.A. Cook, W.J. Kaiser, and E.M. Logothetis. An engine-dynamometer study of the a/f compatibility between a three-way catalyst and an exhaust gas oxygen sensor. SAE paper 830986, 1983.

[12] G.A. Ingram and G. Surnilla. On-line oxygen storage capacity estimation of a catalyst. SAE paper 2003-01-1000, 2003.

[13] E. Shafai, C. Roduner, and H.P. Geering. Indirect adaptive control of a three-way catalyst. SAE paper 961038, 1996.

[14] M.A. Shulman and D.R. Hamburg. Non-ideal properties of ZrO2 and TiO2 exhaust gas oxygen sensors. SAE paper 800018, 1980.

[15] J.R. Theis. Catalytic converter diagnosis using the catalyst exotherm. SAE paper 942058, 1994.

[16] A.T. Vemuri. Diagnosis of sensor bias faults. American Control Conference, 1999.

\section{Figures AND TABles of Experimental Data}

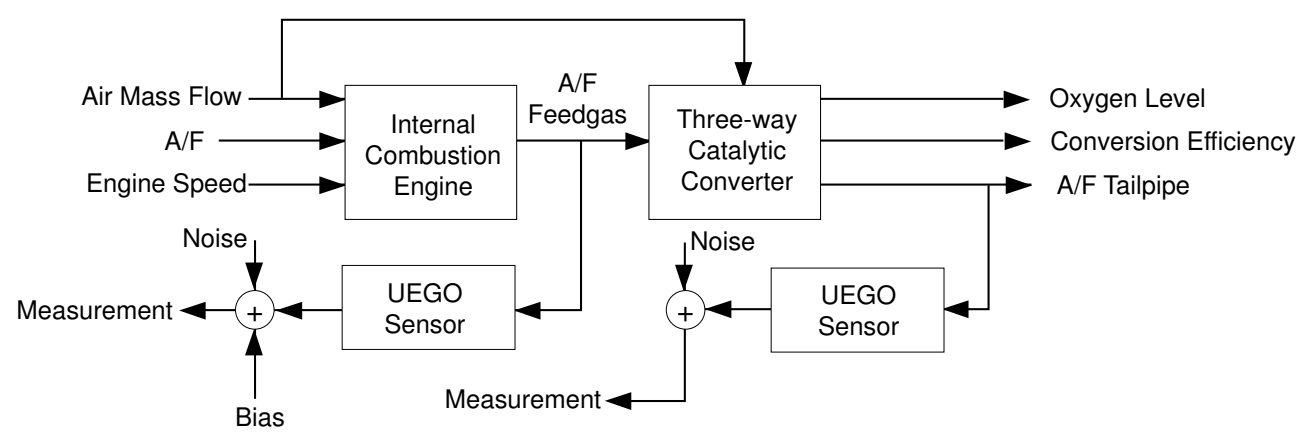

Fig. 1. Engine and Catalyst. Also shown are the upstream and downstream UEGO sensors.

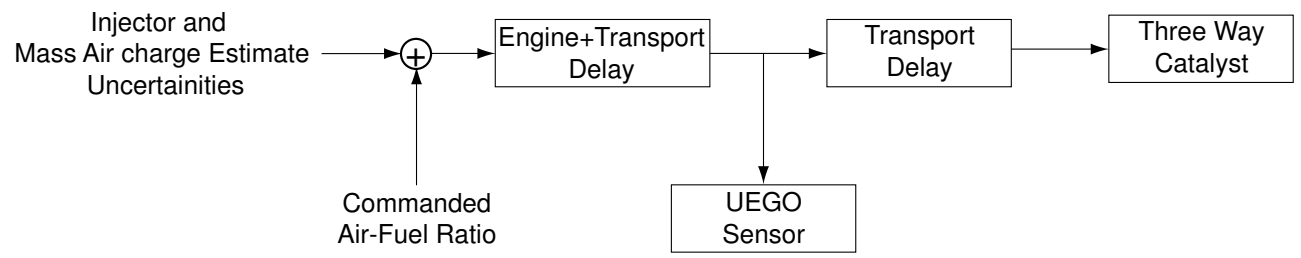

Fig. 2. Simplified engine model. 


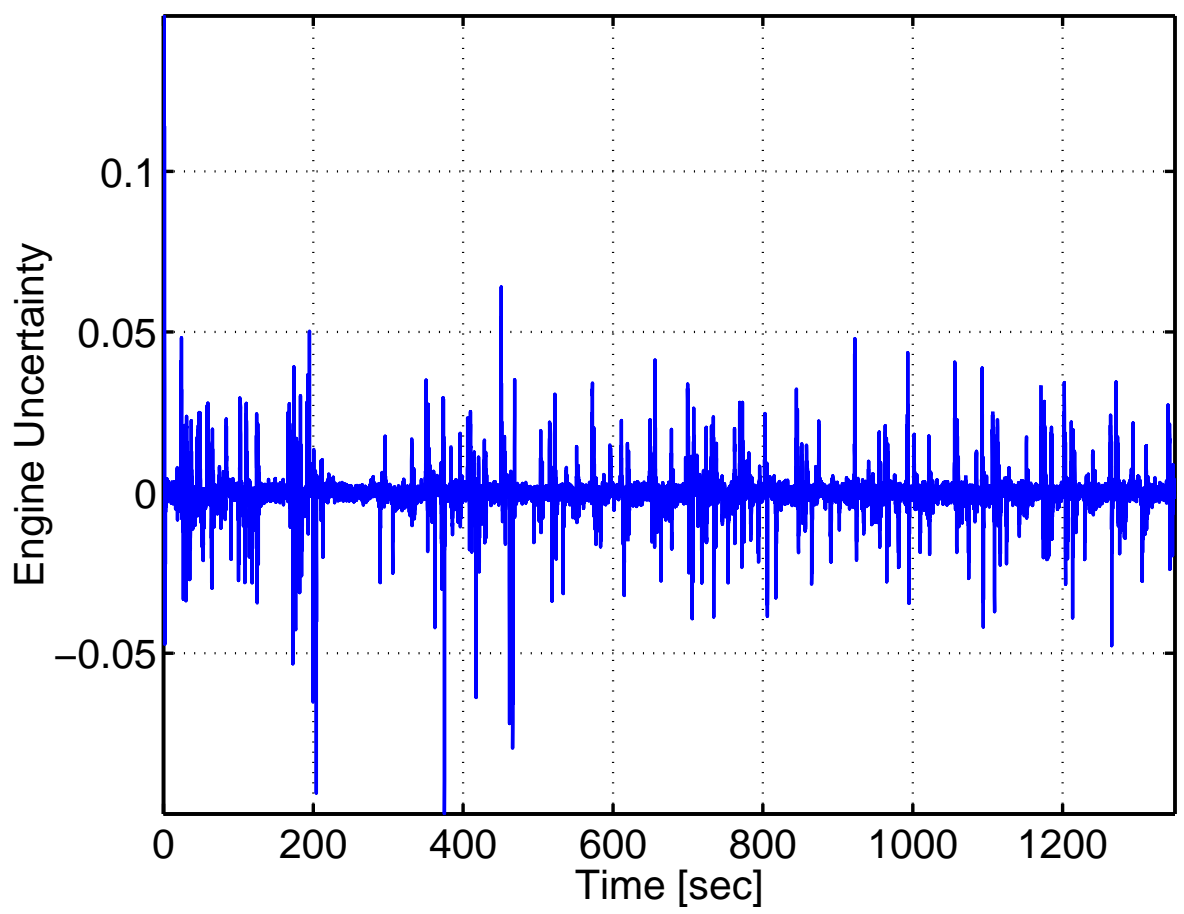

Fig. 3. Estimated uncertainty in the injected fuel quantity and in the estimated air-charge for a $5.4 L$ engine over a drive cycle; units of normalized air-fuel ratio, $\lambda$.

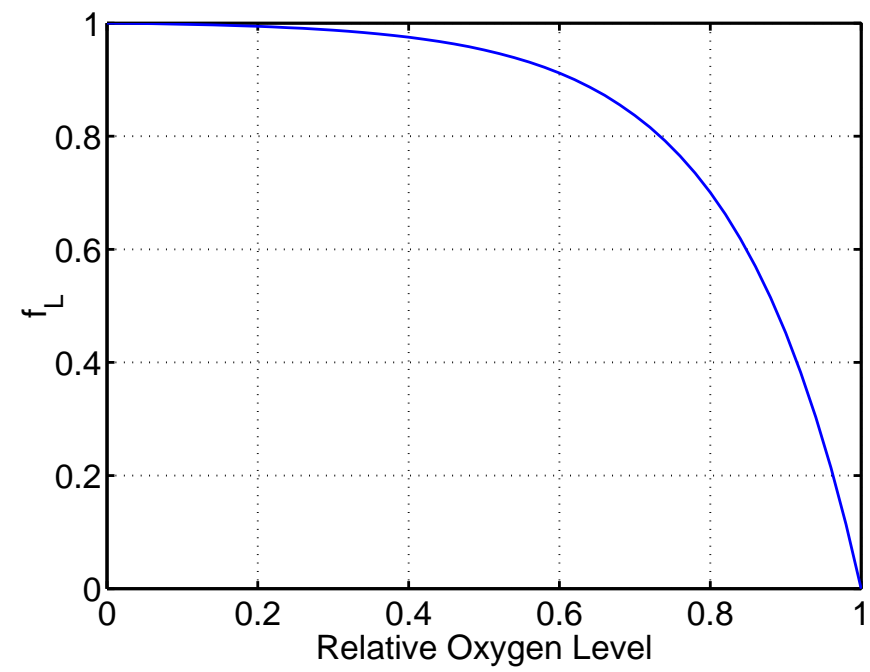

(a)

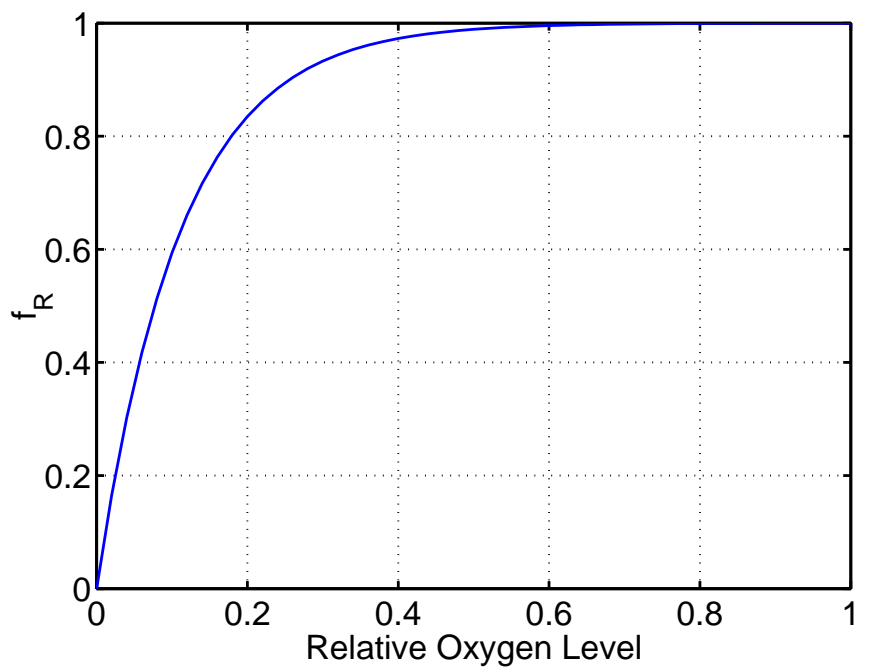

(b)

Fig. 4. (a) Typical function $f_{L}$ (b) Typical function $f_{R}$ 


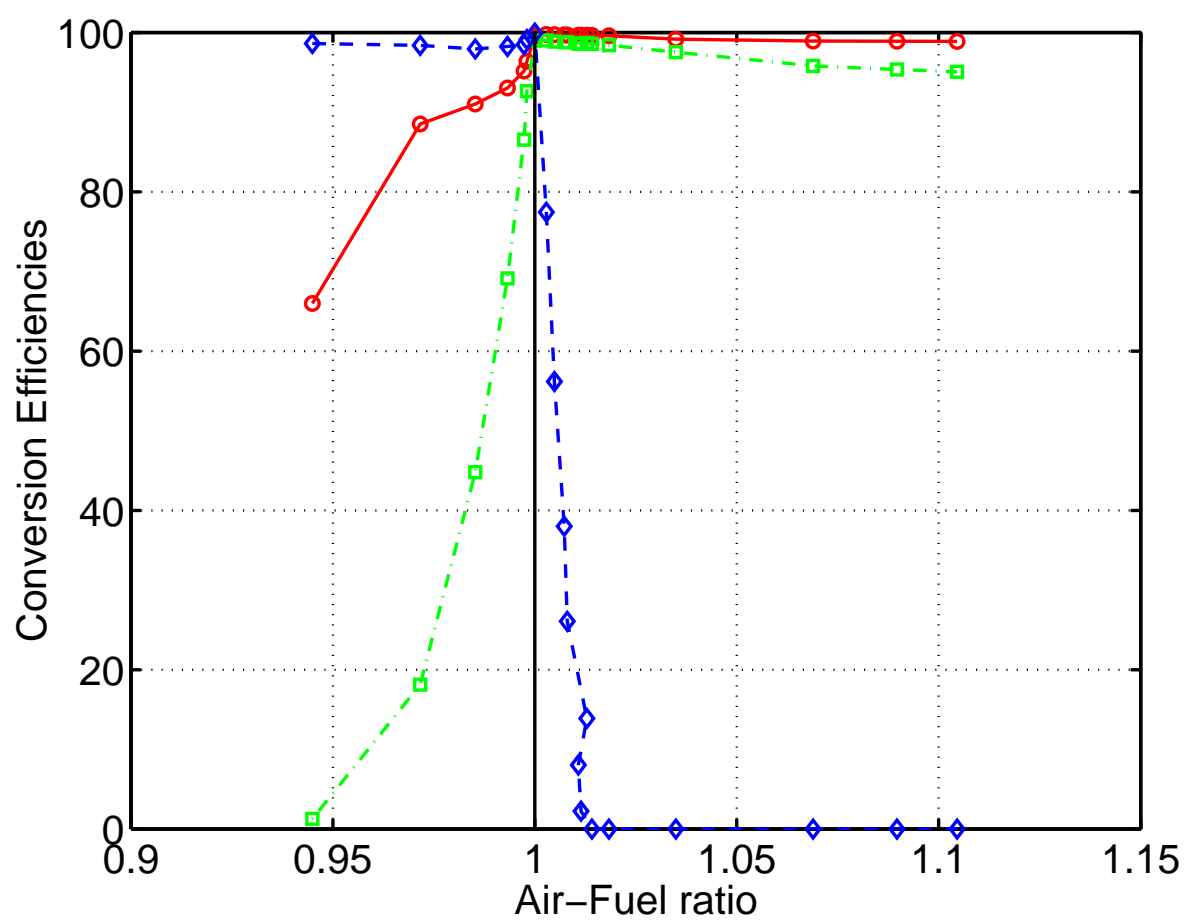

Fig. 5. Conversion efficiencies of $H C$ (solid line) , $C O$ (dot-dashed line), and $N O_{x}$ (dashed line)
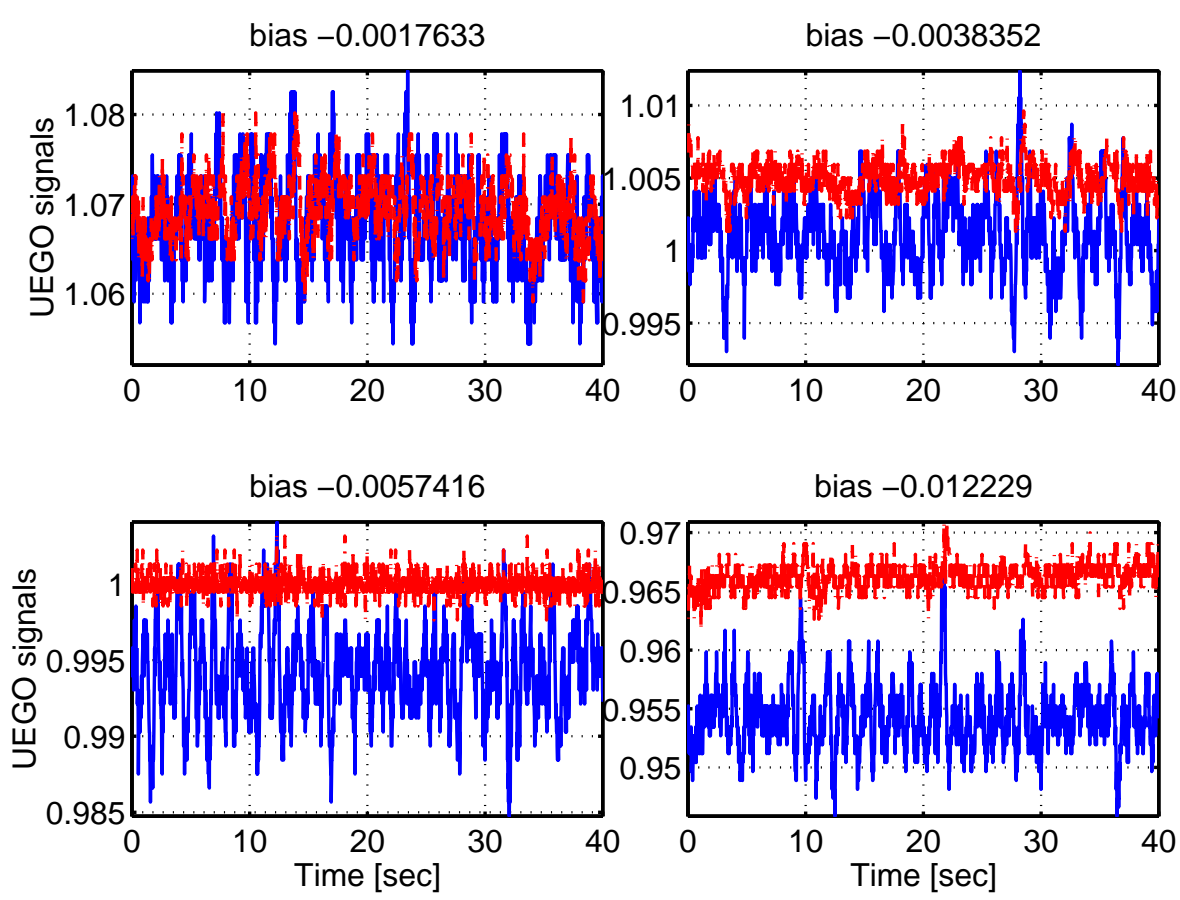

Fig. 6. Upstream (solid line) and downstream (dashed line) sensors output for different values of the air-fuel ratio. The engine speed and the load are constant. 


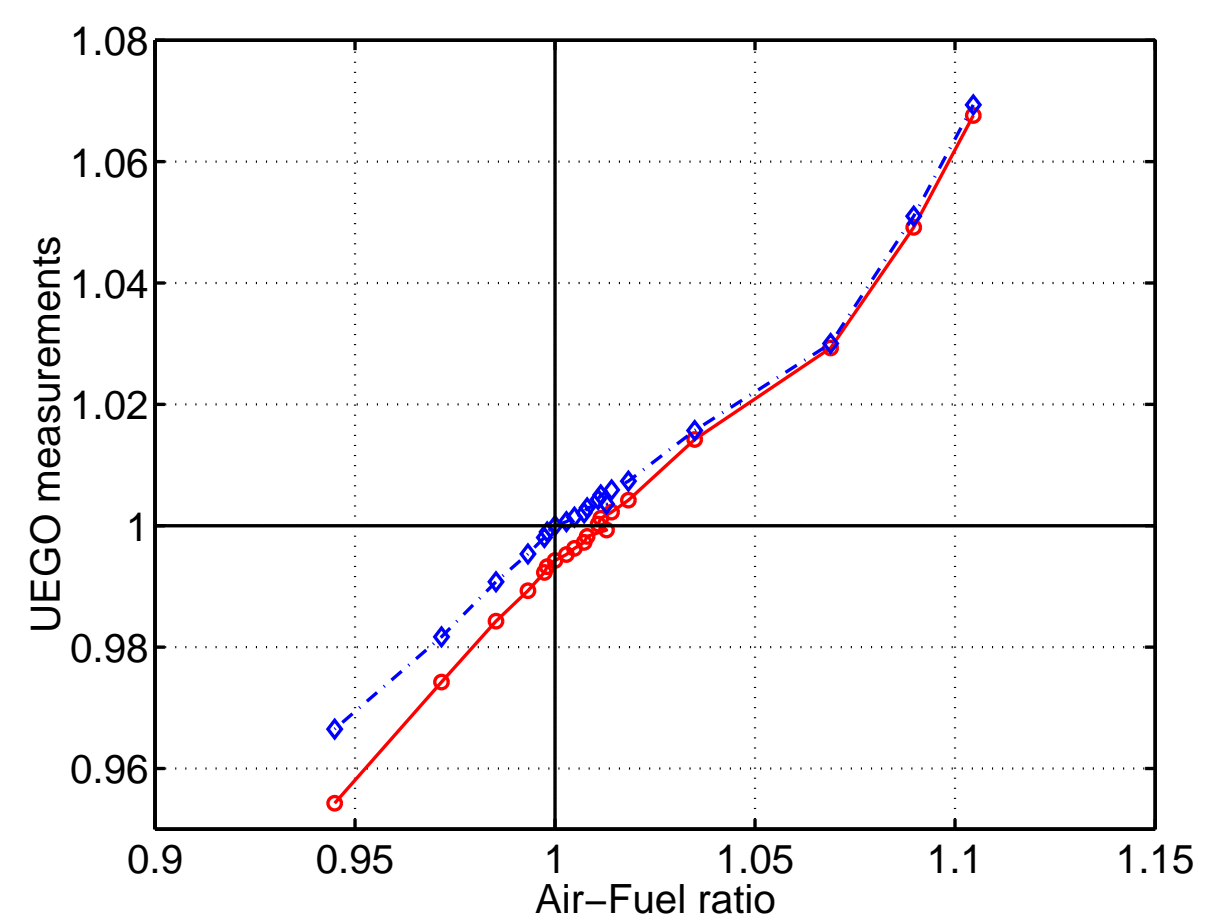

Fig. 7. Feedgas (solid line) and tailpipe (dashed line) UEGO sensors static curves.

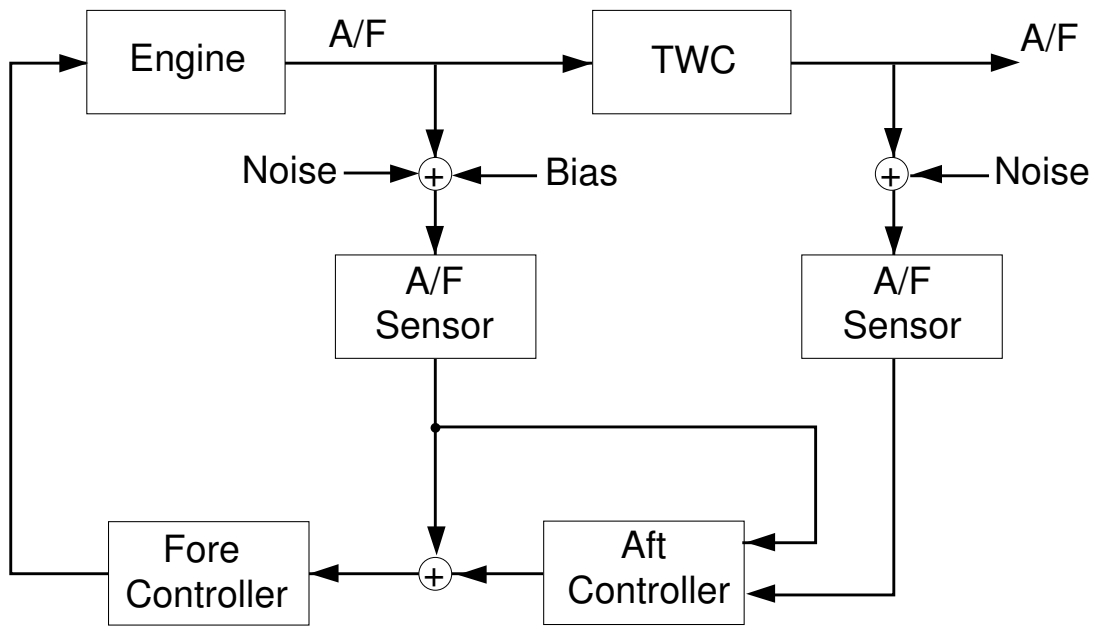

Fig. 8. Dual UEGO Fore-Aft Controller. 

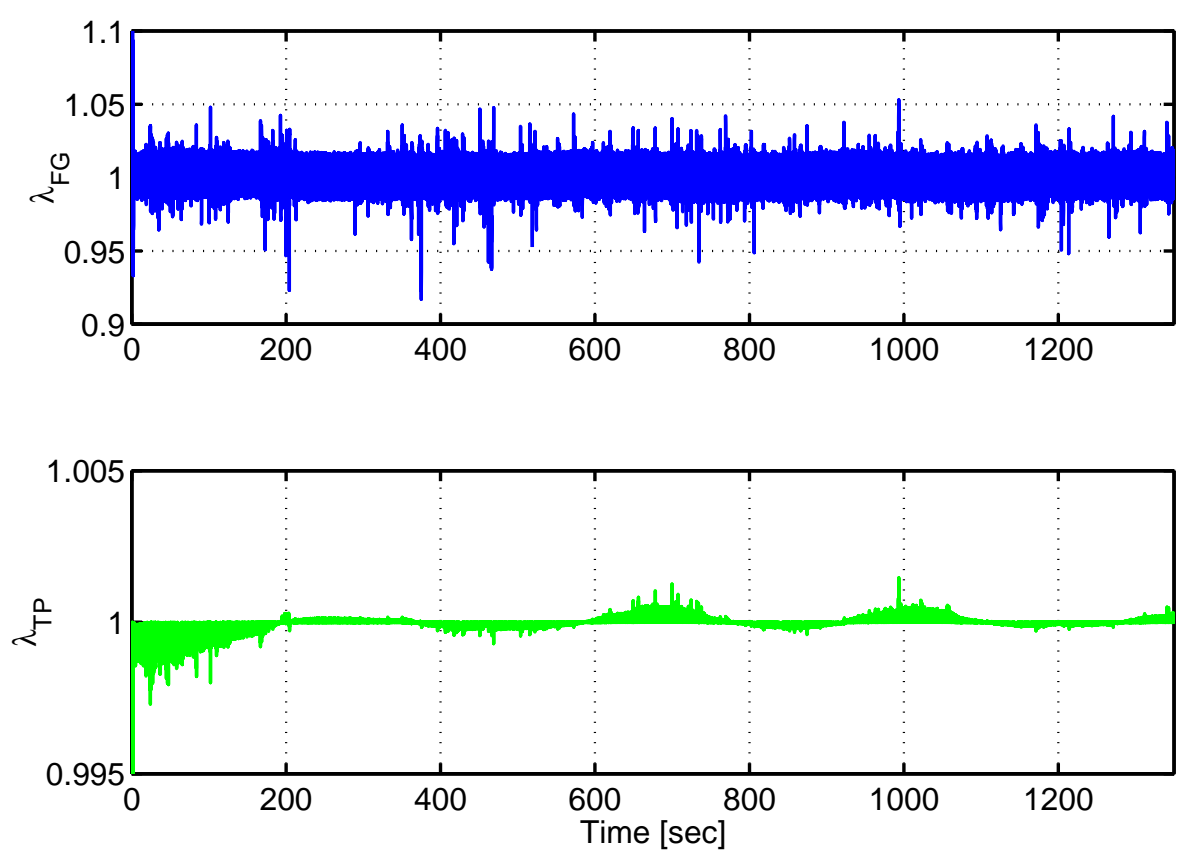

Fig. 9. Air-Fuel ratio at feedgas and tailpipe.
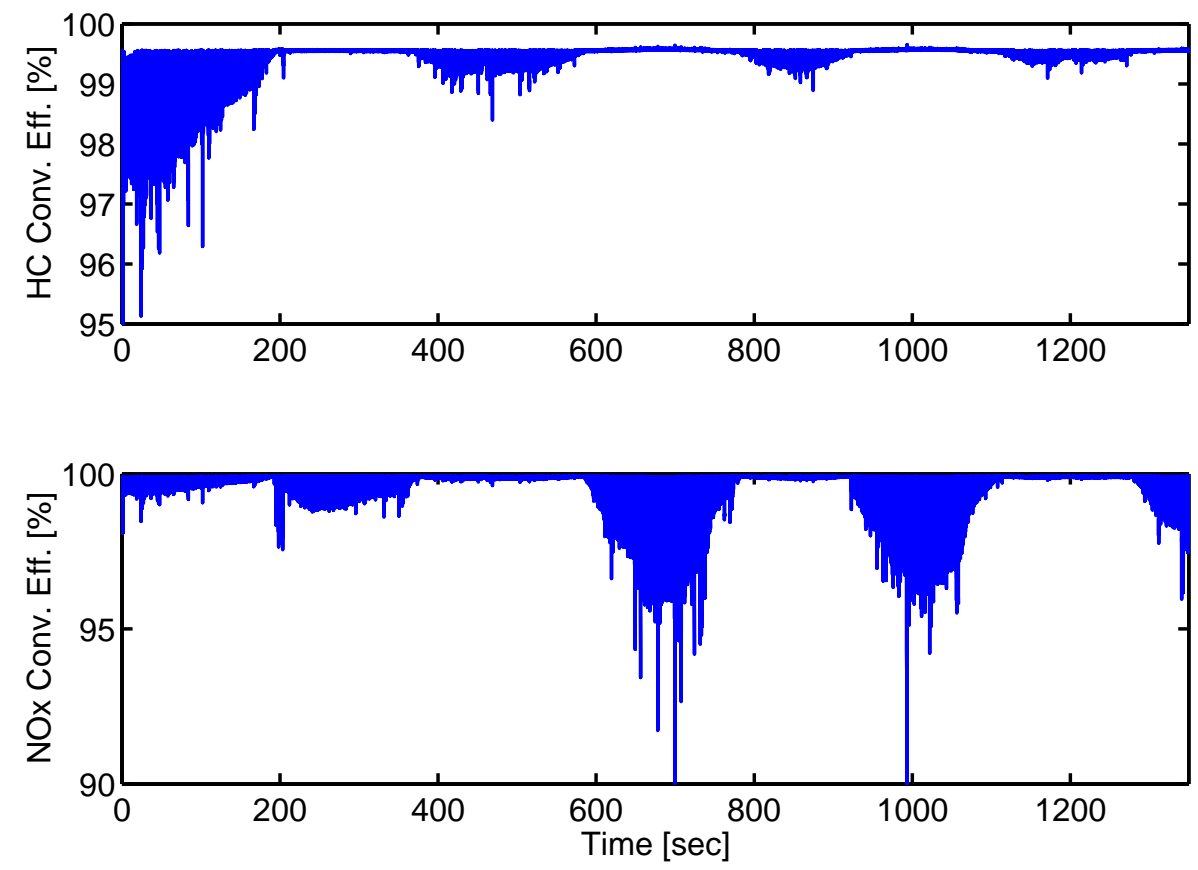

Fig. 10. Conversion efficiencies for unburned hydrocarbons and nitrogen oxide. 

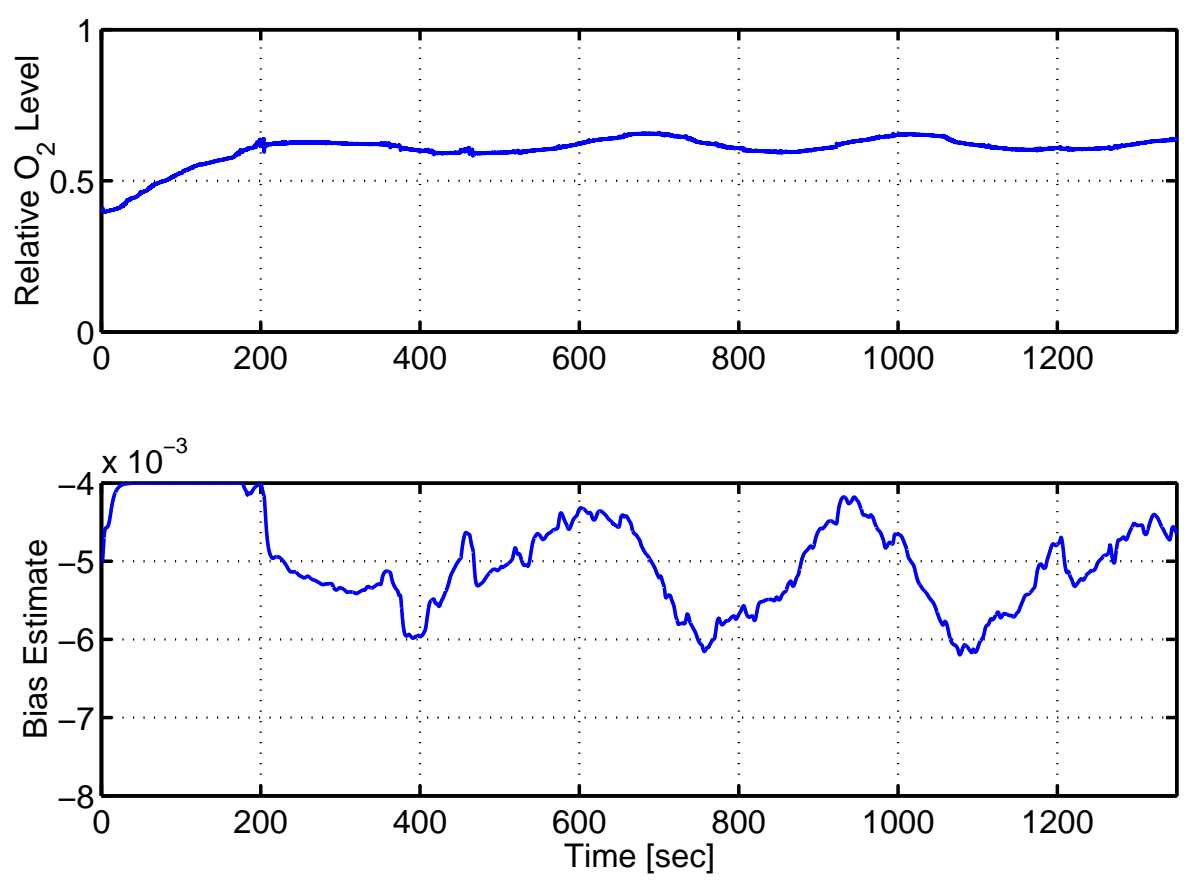

Fig. 11. Relative oxygen level and estimated bias.

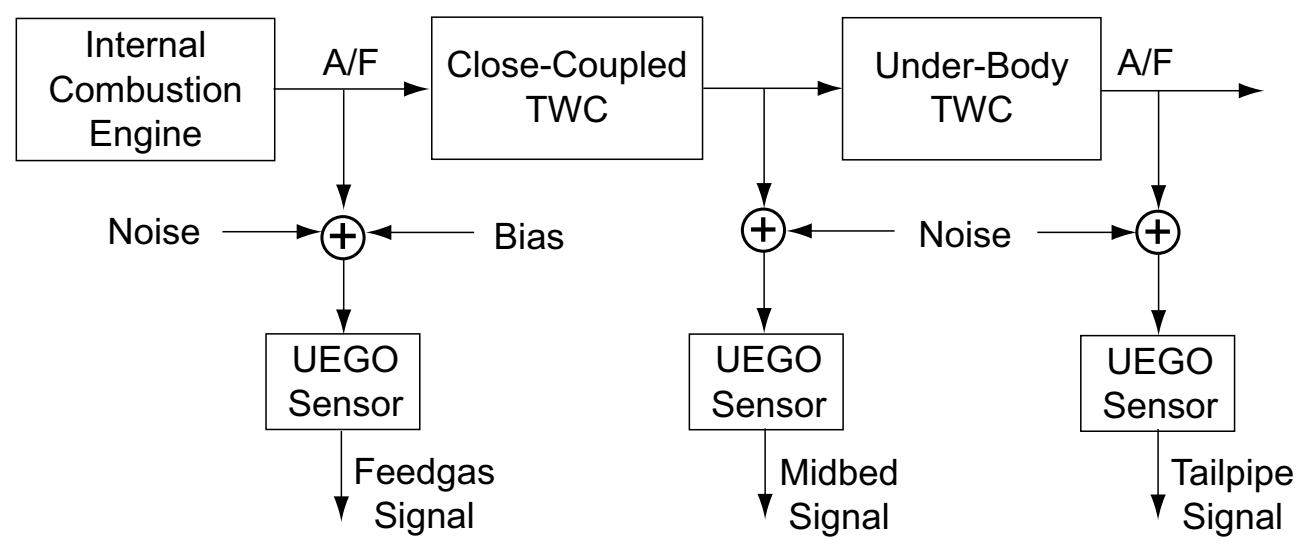

Fig. 12. Configuration with two catalysts. 

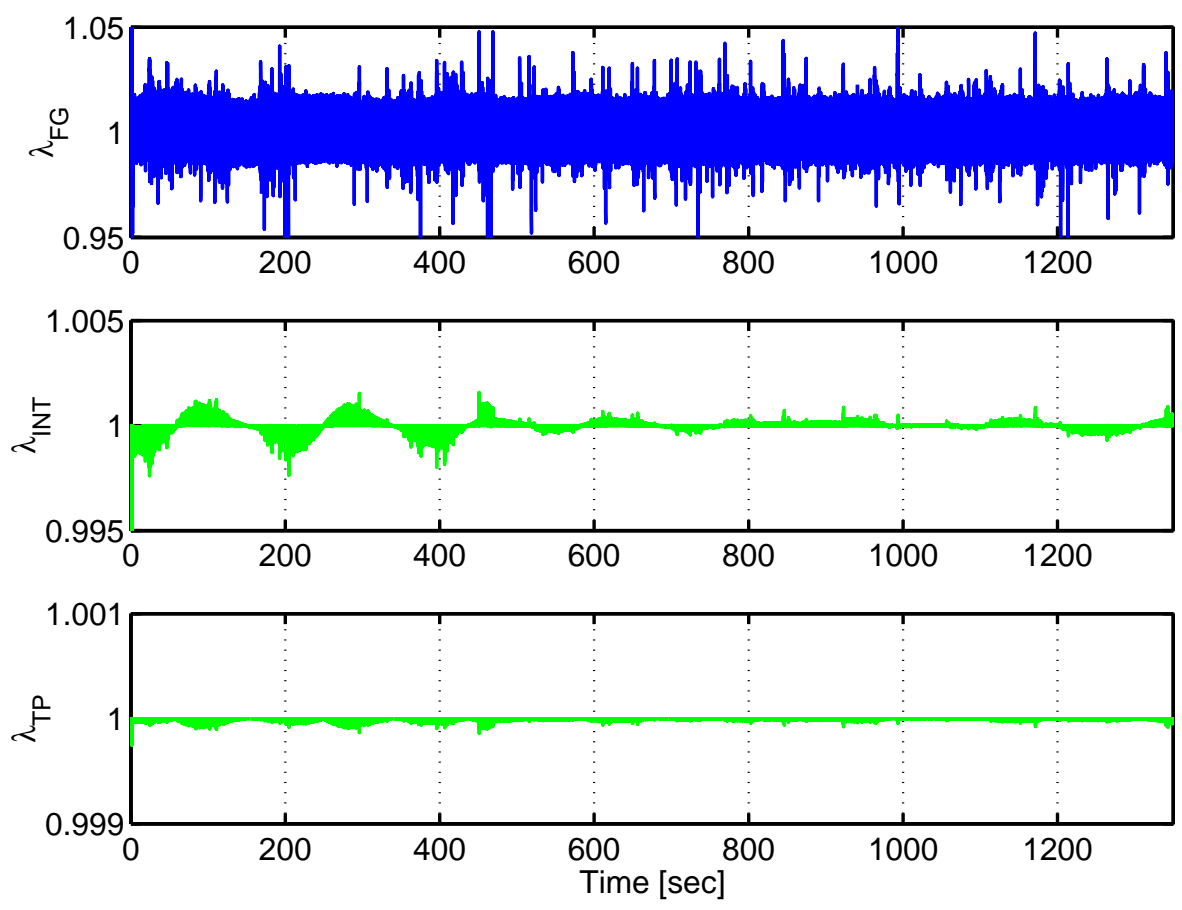

Fig. 13. Air-Fuel ratio respectively at feedgas, after the Close-Coupled TWC and tailpipe.
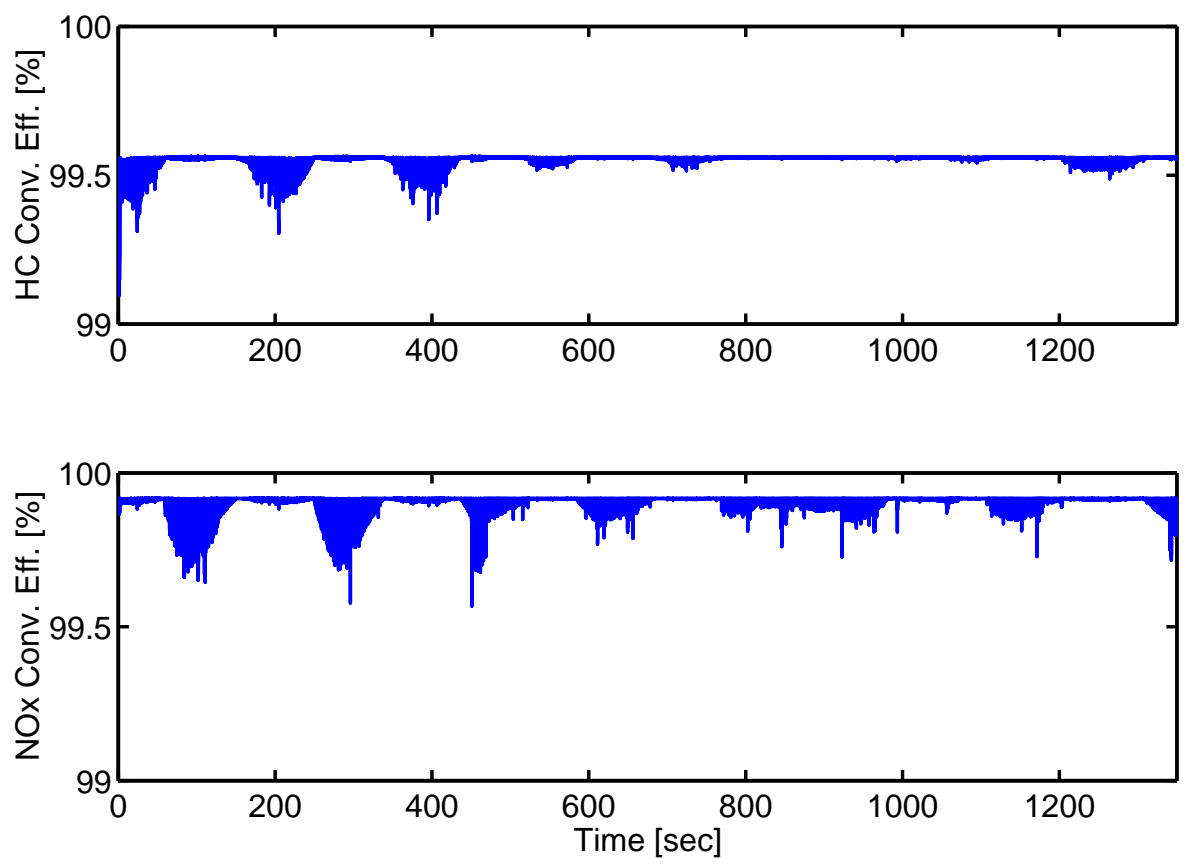

Fig. 14. Conversion efficiencies for unburned hydrocarbons and nitrogen oxide. 

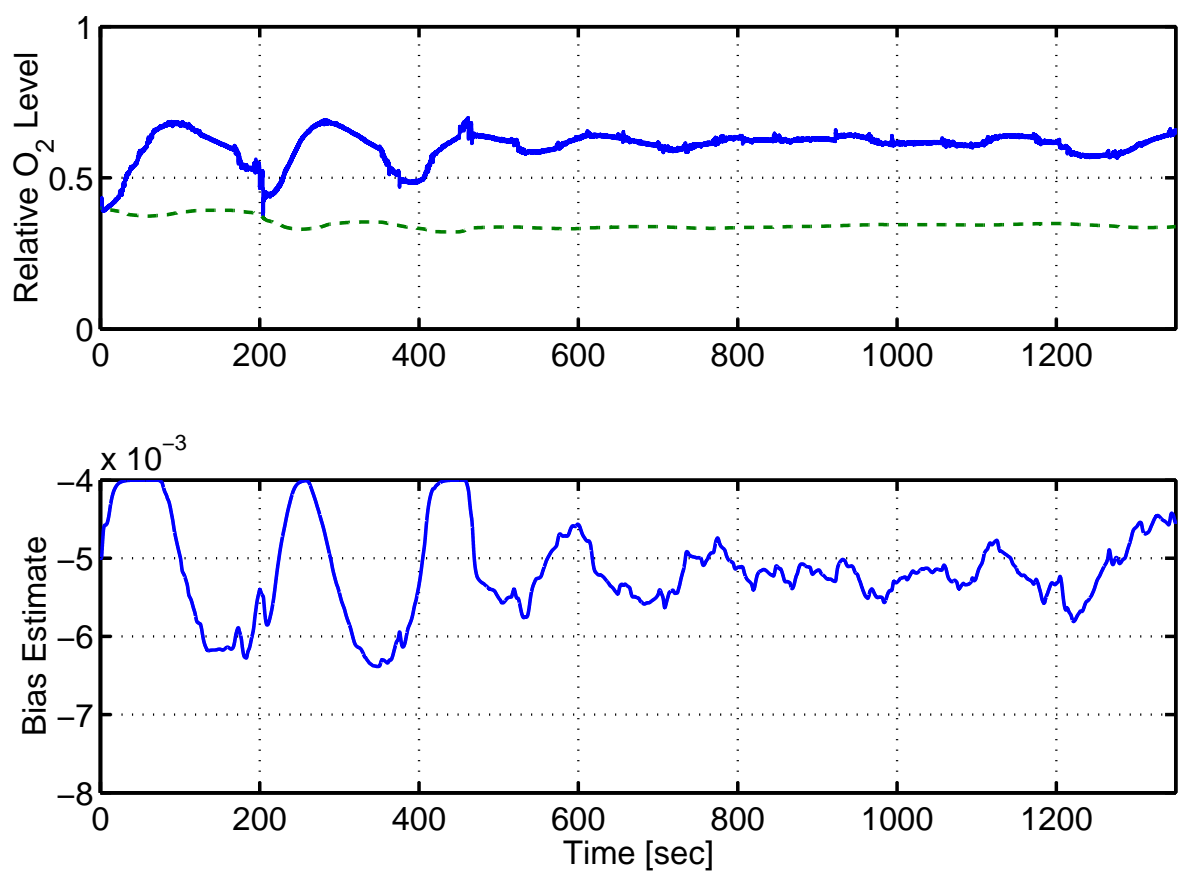

Fig. 15. Relative oxygen level of the CC-TWC (solid line) and the UB-TWC (dashed line), and estimated bias.

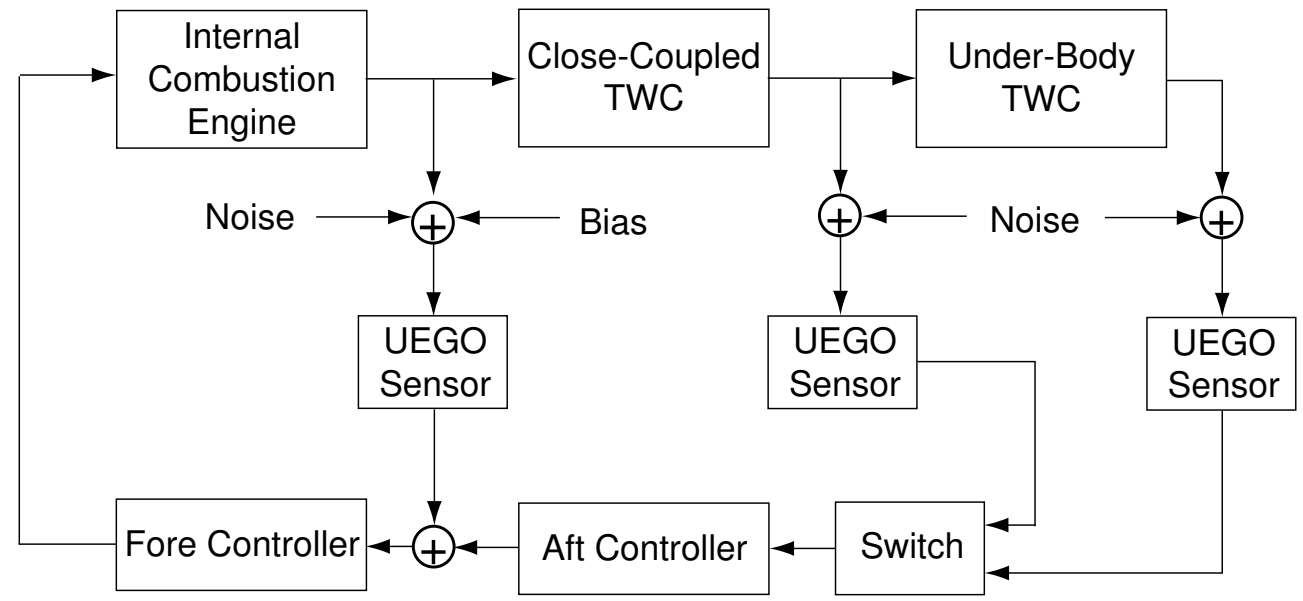

Fig. 16. Block diagram of the system formed by the cascade of the engine and the two catalytic converters regulated by the switching control in series configuration. 


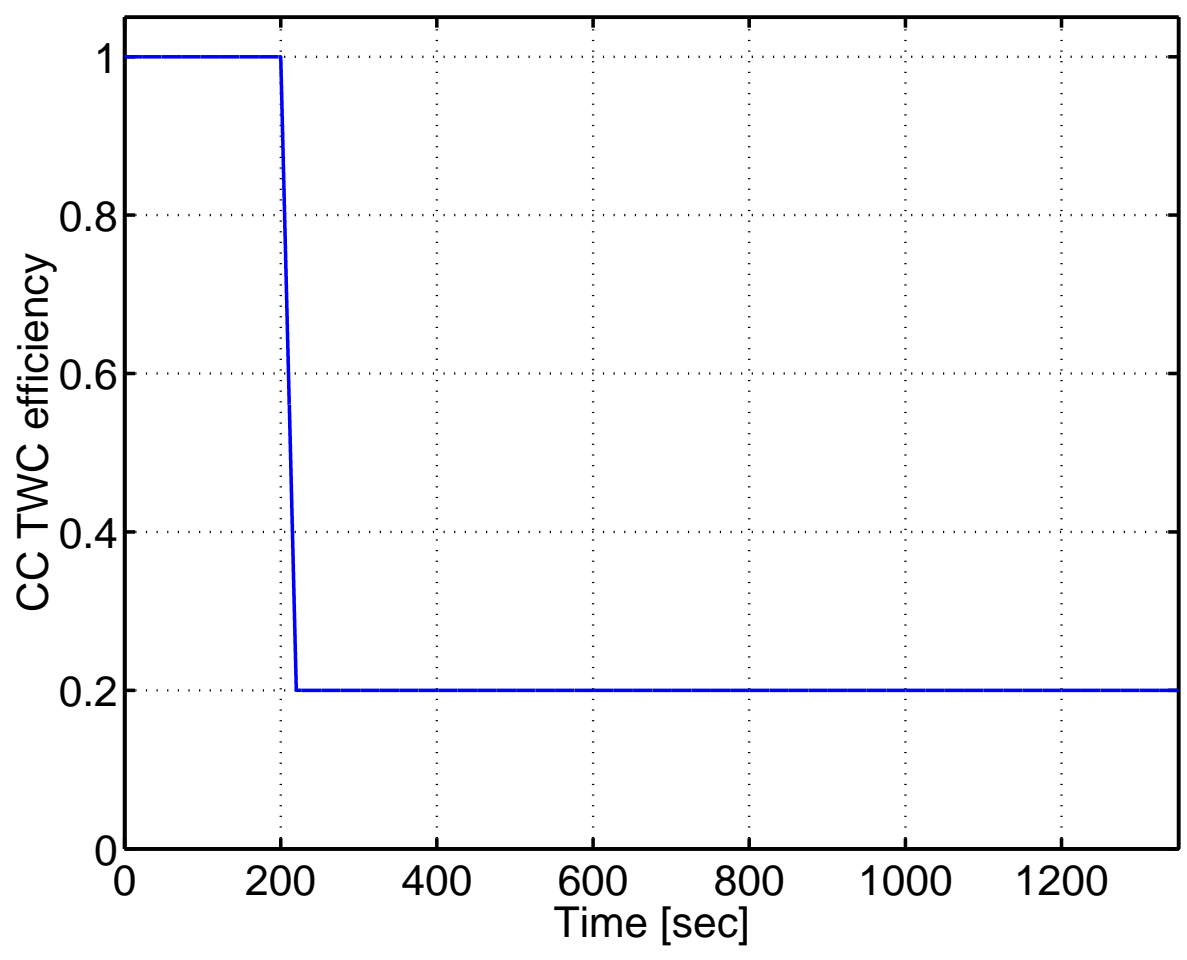

Fig. 17. Efficiency of the Close-Coupled TWC.

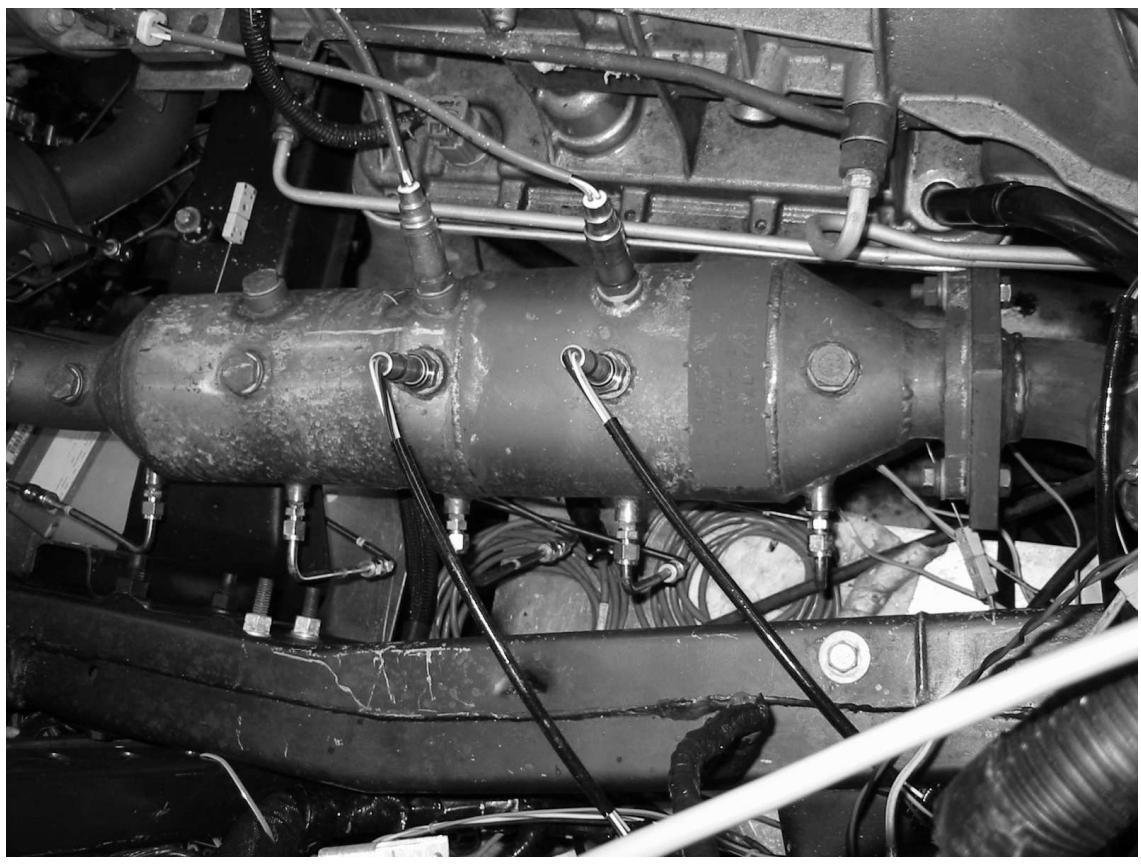

Fig. 18. Photograph of the TWC used on the 5.4L engine, viewed from the top. Feedgas is flowing from right to left. The emissions taps are visible at the bottom of the photograph, the UEGO sensors are in the middle, and the HEGO sensors are toward the top. 


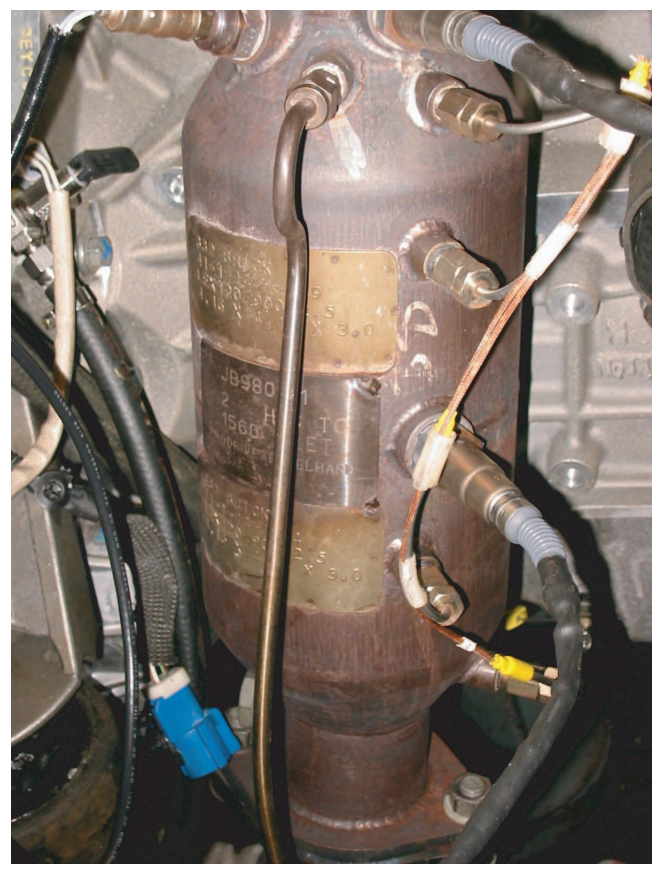

Fig. 19. Photograph of the TWC used on the $2.3 \mathrm{~L}$ engine, viewed from the side. Feedgas is flowing from top to bottom. The feedgas emissions tap is visible at the top center of the photograph, feedgas UEGO and HEGO sensors are at the top left and right, and the brick one UEGO sensors is visible in the middle of the TWC.

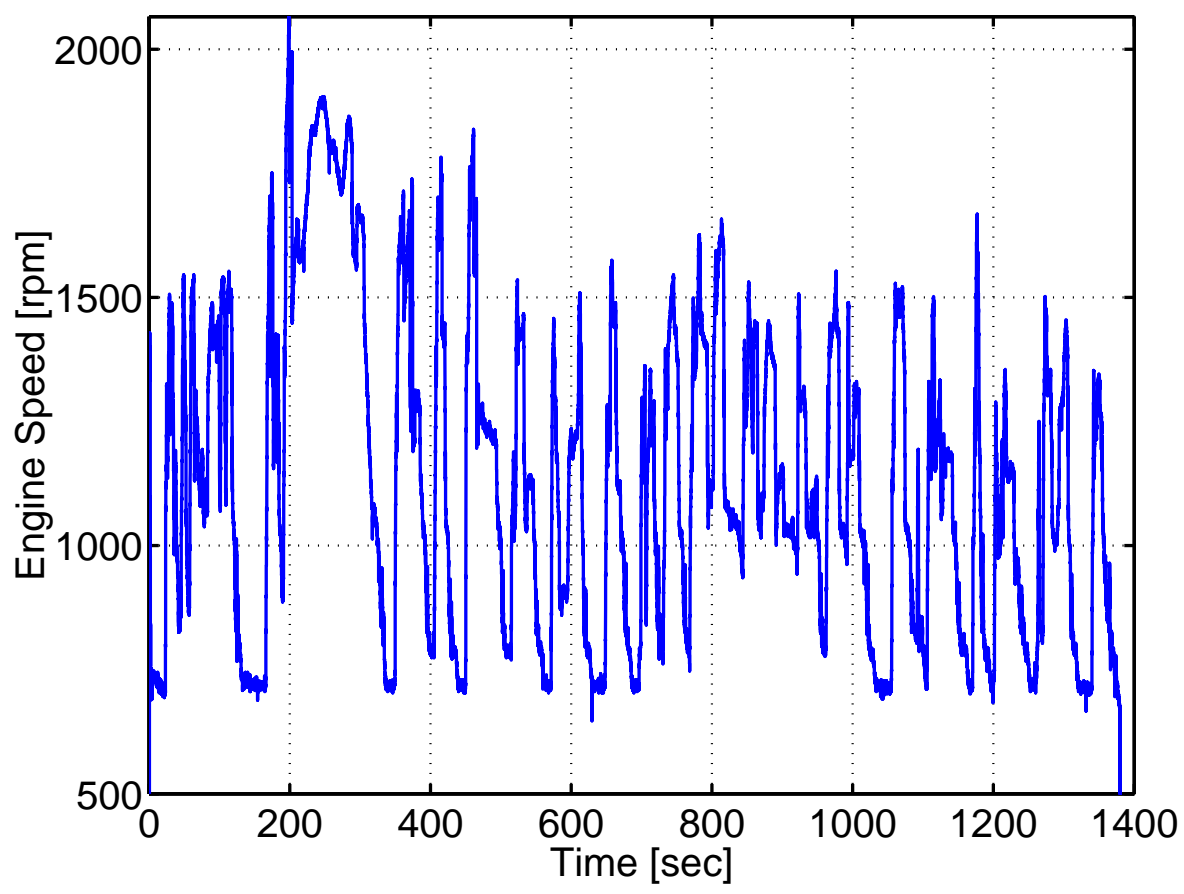

Fig. 20. Engine Speed over Test Cycle. 

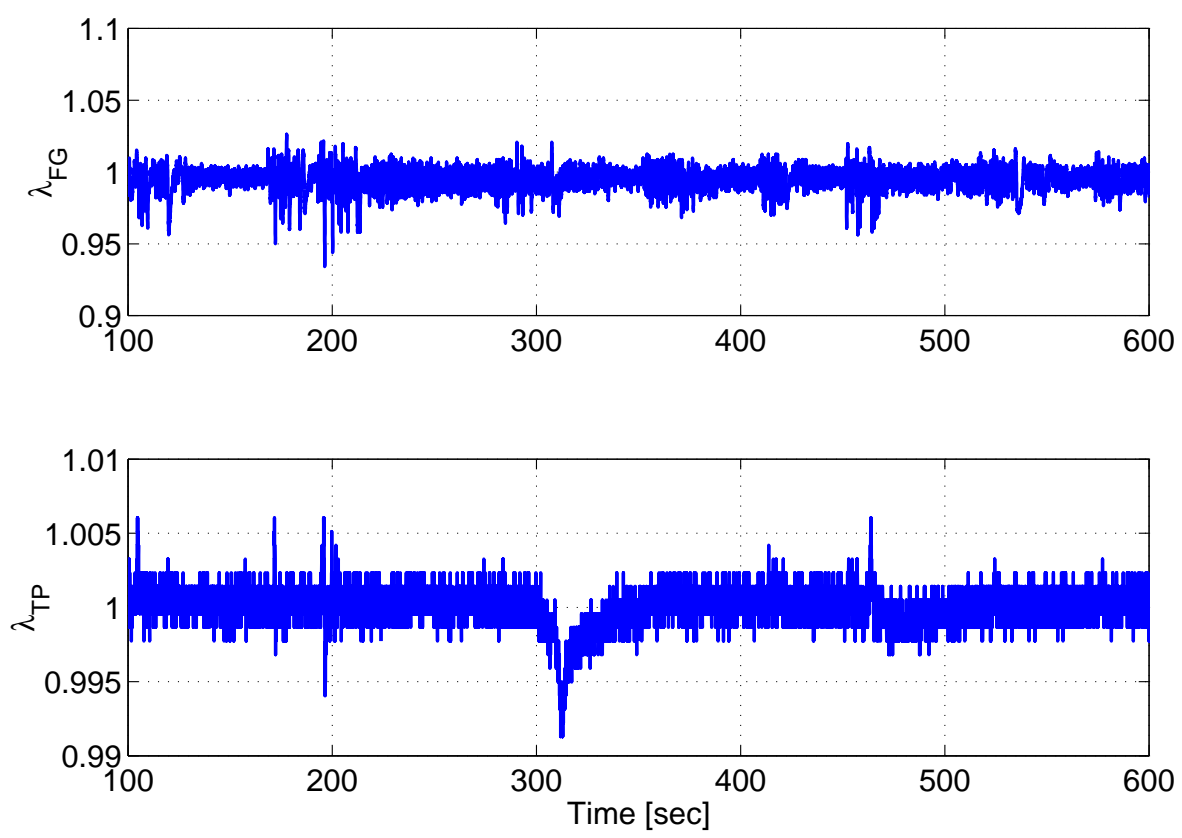

Fig. 21. 5.4L Engine. Air-Fuel ratio at feedgas and tailpipe.
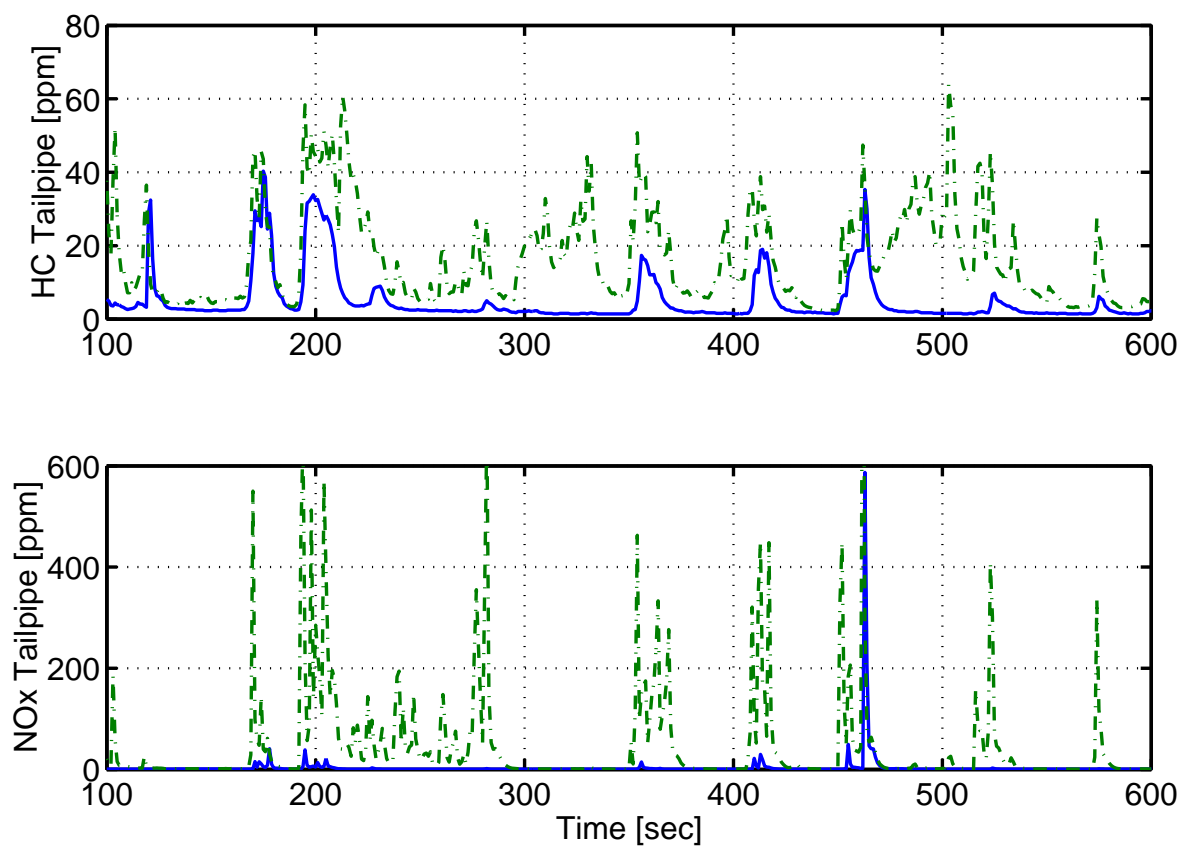

Fig. 22. 5.4L Engine. Post-catalyst $H C$ and $N O x$ emissions in $p p m$ at brick one. The solid line (blue) refers to the dual UEGO controller and the dot-dashed line (green) to the baseline controller. 

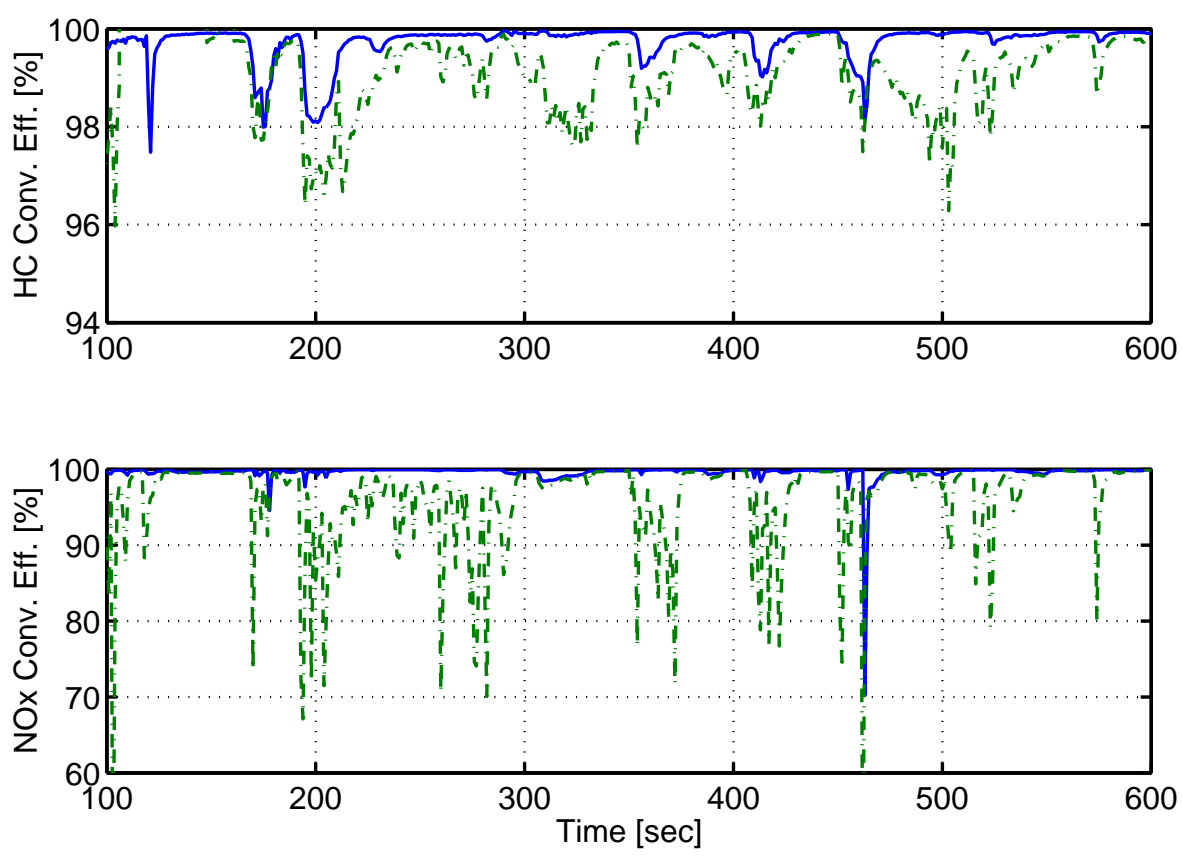

Fig. 23. $5.4 L$ Engine. Instantaneous $H C$ and $N O x$ conversion efficiencies at brick one. The solid line (blue) refers to the dual UEGO controller and the dot-dashed line (green) to the baseline controller.
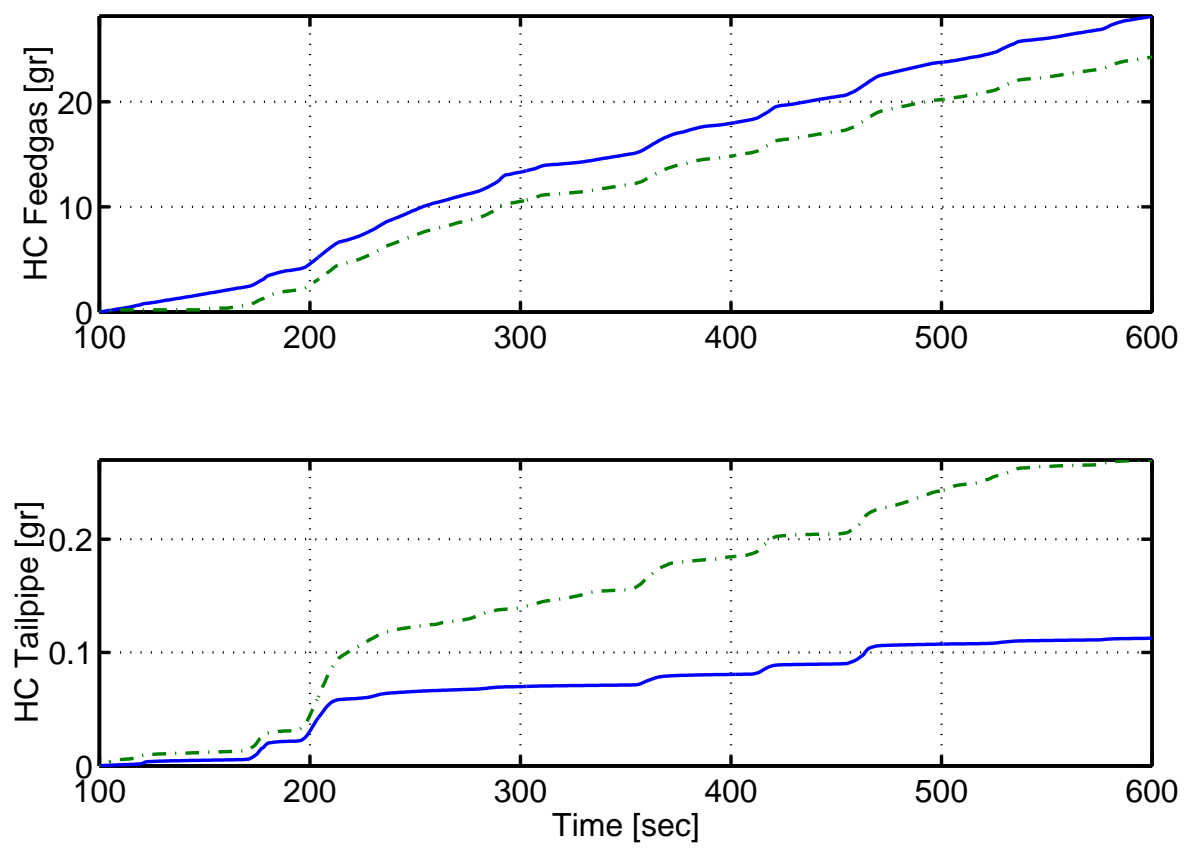

Fig. 24. 5.4L Engine. Pre- and Post-catalyst cumulative $H C$ emissions in grams at brick one. The solid line (blue) refers to the dual UEGO controller and the dot-dashed line (green) to the baseline controller. 

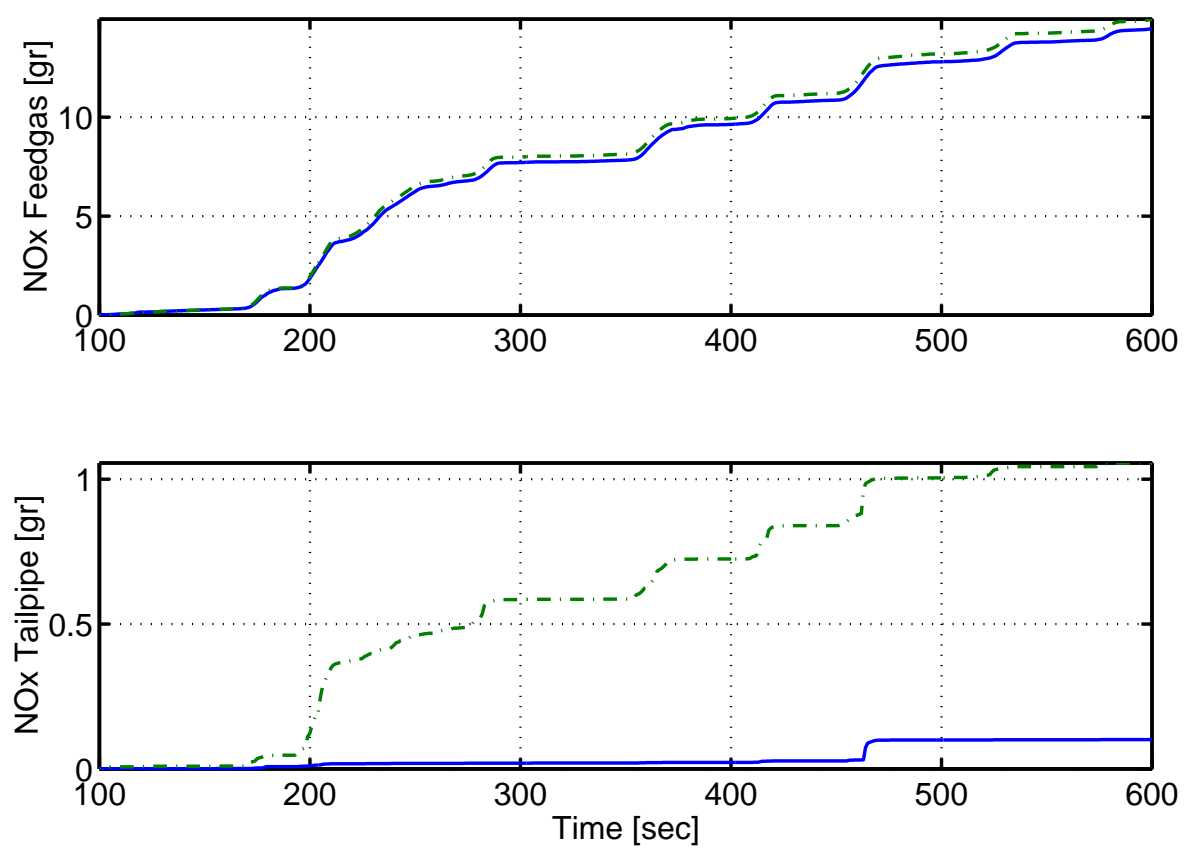

Fig. 25. 5.4L Engine. Pre- and Post-catalyst cumulative NOx emissions in grams at brick one. The solid line (blue) refers to the dual UEGO controller and the dot-dashed line (green) to the baseline controller.

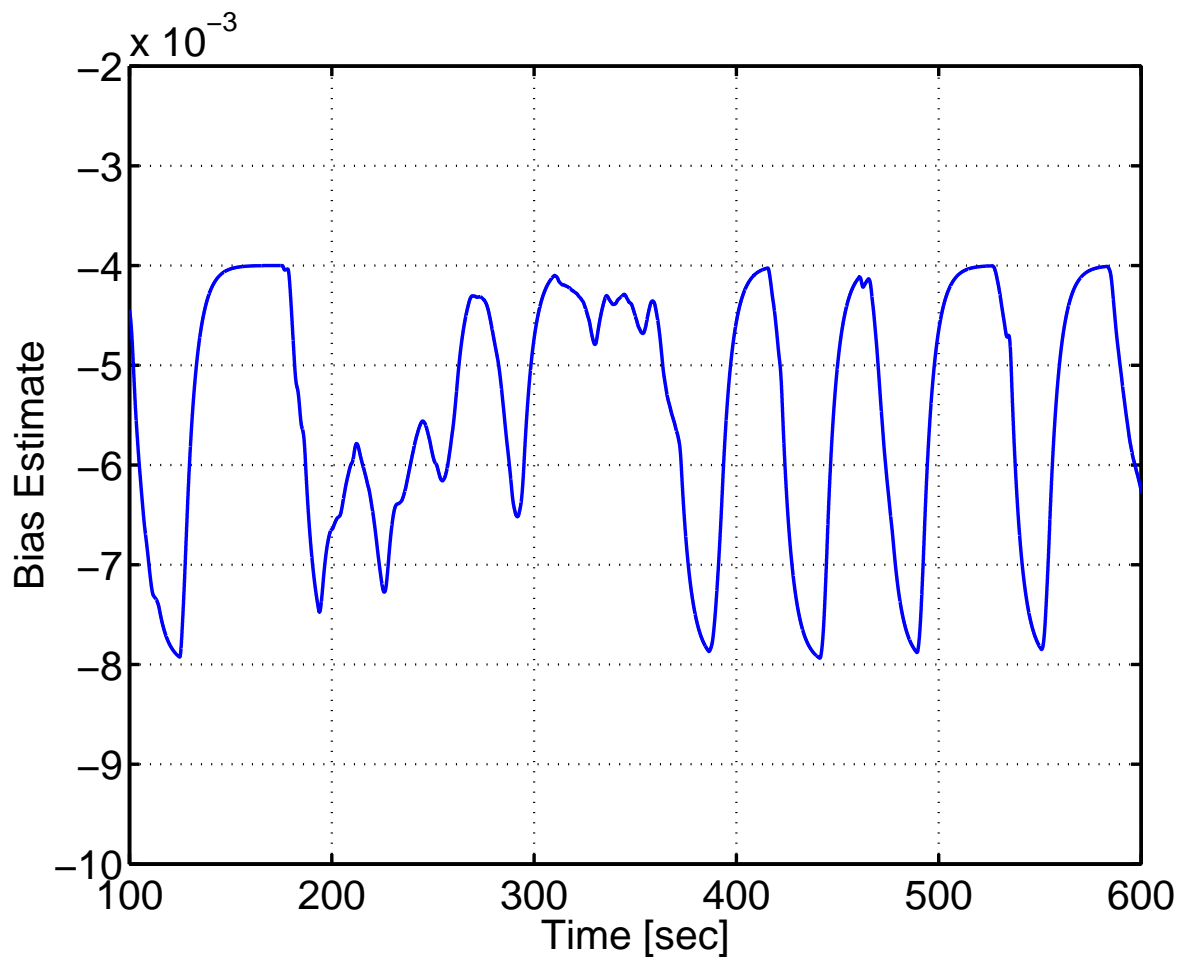

Fig. 26. 5.4L Engine. Bias estimate. Typical trace from 100 to 600 seconds when the UEGO is positioned at brick one. 


\section{TABLE VII}

5.4L Engine. Emission RESUlts From 100 to 600 SECONDS of the FTP CyCle, With tailpipe EMissions COLLECTED AT BRICK ONE. IN A), THE COMPLETE CONTROL ALGORITHM WAS IMPLEMENTED; IN B), BIAS ESTIMATION WAS USED BUt THE PROPORTIONAL ACTION IN THE AFT CONTROLLER WAS DiSABlED. DUAL-UEGO TAILPIPE $A / F$ WAS MEASURED AT BRICK ONE.

\begin{tabular}{c||c|cc||c|cc}
\multirow{2}{*}{ Controller } & \multicolumn{3}{c||}{ HC } & \multicolumn{3}{c}{ NOx } \\
\cline { 2 - 7 } & Conv. eff. [\%] & Tot. FG $[g r]$ & Tot. TP $[g r]$ & Conv. eff. [\%] & Tot. FG $[g r]$ & Tot. TP $[g r]$ \\
\hline \hline Baseline & 99.04 & 24.27 & 0.2701 & 93.89 & 14.96 & 1.0559 \\
& & & & & & \\
Dual UEGO $^{a}$ & 99.76 & 28.15 & 0.1127 & 99.53 & 14.49 & 0.1009 \\
Dual UEGO $^{b}$ & 99.35 & 26.69 & 0.2071 & 98.80 & 15.06 & 0.2591
\end{tabular}

TABLE VIII

5.4L Engine. Emission RESults From 100 to 600 SECONDS of the FTP CyCle, With tailpipe EMissions COLLECTED AT BRICK TWO. IN A), THE COMPLETE CONTROL ALGORITHM WAS IMPLEMENTED; IN B), BIAS ESTIMATION WAS USED BUT THE PROPORTIONAL ACTION IN THE AFT CONTROLLER WAS DISABLED; IN C), THE BIAS ESTIMATOR WAS DISABLED DURING TRANSIENTS (SEE [7]), BUT OTHERWISE, THE COMPLETE CONTROL ALGORITHM

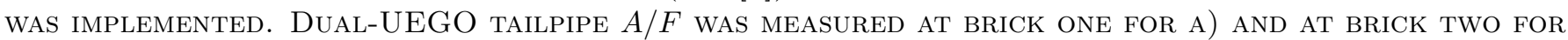
B) AND C).

\begin{tabular}{c||c|cc||c|cc}
\multirow{2}{*}{ Controller } & \multicolumn{3}{c||}{ HC } & \multicolumn{3}{c}{ NOx } \\
\cline { 2 - 7 } & Conv. eff. [\%] & Tot. FG $[g r]$ & Tot. TP $[g r]$ & Conv. eff. [\%] & Tot. FG $[g r]$ & Tot. TP $[g r]$ \\
\hline \hline Baseline & 99.90 & 25.43 & 0.0268 & 98.99 & 17.45 & 0.3002 \\
Baseline & 94.57 & not. av. & not.av. & 97.75 & not.av. & not. av. \\
& & & & & & \\
Dual UEGO $^{a}$ & 99.98 & 25.91 & 0.0043 & 99.90 & 14.17 & 0.0099 \\
Dual UEGO $^{a}$ & 99.92 & 26.91 & 0.0230 & 99.93 & 18.40 & 0.0088 \\
\hline Dual UEGO $^{b}$ & 99.91 & 26.86 & 0.0311 & 99.72 & 15.45 & 0.0460 \\
Dual UEGO $^{c}$ & 99.92 & 24.03 & 0.0224 & 99.50 & 15.90 & 0.0805 \\
Dual UEGO $^{c}$ & 99.94 & 26.49 & 0.0176 & 99.23 & 16.55 & 0.1439 \\
Dual UEGO $^{c}$ & 99.89 & 27.72 & 0.0308 & 99.51 & 16.90 & 0.0754 \\
Dual UEGO $^{c}$ & 99.70 & 28.98 & 0.0926 & 99.61 & 16.62 & 0.0358 \\
Dual UEGO $^{c}$ & 99.91 & 28.53 & 0.0268 & 98.52 & 17.73 & 0.2258
\end{tabular}

TABLE IX

5.4L Engine. EMission ReSUlts From 100 to 1400 SECONDS of the FTP CyCle, With tailpipe EMissions COLLECTED AT BRICK TWO. IN A), THE COMPLETE CONTROL ALGORITHM WAS IMPLEMENTED; IN B), BIAS ESTIMATION WAS USED BUt THE PROPORTIONAL ACTION IN THE AFT CONTROLLER WAS DiSABled. DUAL-UEGO TAILPIPE $A / F$ WAS MEASURED AT BRICK ONE FOR A) AND AT BRICK TWO FOR B).

\begin{tabular}{c||c|cc||c|cc}
\multirow{2}{*}{ Controller } & \multicolumn{4}{c||}{ HC } & \multicolumn{3}{c}{ NOx } \\
\cline { 2 - 7 } & Conv. eff. [\%] & Tot. FG $[\mathrm{gr}]$ & Tot. TP $[\mathrm{gr}]$ & Conv. eff. [\%] & Tot. FG $[\mathrm{gr}]$ & Tot. TP $[\mathrm{gr}]$ \\
\hline \hline Baseline & 99.91 & 56.69 & 0.0560 & 99.10 & 32.59 & 0.4106 \\
& & & & & & \\
Dual UEGO $^{a}$ & 99.98 & 56.31 & 0.0091 & 99.89 & 25.55 & 0.0221 \\
Dual UEGO $^{b}$ & 99.54 & not. av. & not.av. & 99.55 & not.av. & not. av.
\end{tabular}


TABLE X

2.3L Engine. Emission Results from 100 to 1400 Seconds of the FTP CyCle, With tailpipe emissions collected at Brick two. Dual-UEGo tailpipe $A / F$ was measured at brick one. In a) the UEGO SENSORS IN FEEDGAS AND TAILPIPE WERE FROM TWO DIFFERENT MANUFACTURERS; IN B) BOTH SENSORS WERE FROM THE SAME MANUFACTURER

\begin{tabular}{c||c|cc||c|cc}
\multirow{2}{*}{ Controller } & \multicolumn{5}{c||}{ HC } & \multicolumn{3}{c}{ NOx } \\
\cline { 2 - 7 } & Conv. eff. [\%] & Tot. FG [gr] & Tot. TP [gr] & Conv. eff. [\%] & Tot. FG [gr] & Tot. TP $[g r]$ \\
\hline \hline Baseline & 98.58 & 31.19 & 0.5433 & 99.95 & 14.87 & 0.0149 \\
& & & & & & \\
Dual UEGO $^{a}$ & 99.83 & 27.59 & 0.0837 & 99.73 & 20.68 & 0.1437 \\
Dual UEGO $^{a}$ & 99.90 & 27.52 & 0.0500 & 99.68 & 20.65 & 0.0648 \\
Dual UEGO $^{a}$ & 99.84 & 28.99 & 0.0753 & 99.95 & 21.49 & 0.0109 \\
& & & & & & \\
Dual UEGO $^{b}$ & 99.51 & 28.07 & 0.2031 & 99.94 & 28.57 & 0.0308 \\
Dual UEGO $^{b}$ & 99.73 & 27.75 & 0.1276 & 99.92 & 29.40 & 0.0336 \\
Dual UEGO $^{b}$ & 99.68 & 29.21 & 0.1437 & 99.77 & 24.44 & 0.0412
\end{tabular}

TABLE XI

2.3L Engine. EMission Results from 100 to 1400 SECONDS of the FTP CyCle, With tailpipe EMissions Collected at Brick two. Dual-UEGo tailpipe $A / F$ was measured at Brick two. The UEGO Sensors in FEEDGAS AND TAILPIPE WERE FROM TWO DIFFERENT MANUFACTURERS;

\begin{tabular}{c||c|cc||c|cc}
\multirow{2}{*}{ Controller } & \multicolumn{3}{c||}{ HC } & \multicolumn{3}{c}{ NOx } \\
\cline { 2 - 7 } & Conv. eff. [\%] & Tot. FG $[g r]$ & Tot. TP $[g r]$ & Conv. eff. [\%] & Tot. FG $[g r]$ & Tot. TP $[g r]$ \\
\hline \hline Baseline & 98.58 & 31.19 & 0.5433 & 99.95 & 14.87 & 0.0149 \\
& & & & & & \\
Dual UEGO & 99.91 & 27.22 & 0.0358 & 99.85 & 22.86 & 0.0364 \\
Dual UEGO & 99.81 & 28.19 & 0.0921 & 98.27 & 20.25 & 0.3087 \\
Dual UEGO & 99.72 & 27.41 & 0.1127 & 99.85 & 23.19 & 0.0474 \\
Dual UEGO & 99.87 & 29.85 & 0.0663 & 98.84 & 8.01 & 0.1333 \\
Dual UEGO & 99.11 & 29.66 & 0.3244 & 99.85 & 20.59 & 0.0440 \\
Dual UEGO & 99.71 & 28.00 & 0.0906 & 99.70 & 18.56 & 0.0637 \\
Dual UEGO & 99.87 & 27.71 & 0.0663 & 99.69 & 18.89 & 0.0581
\end{tabular}



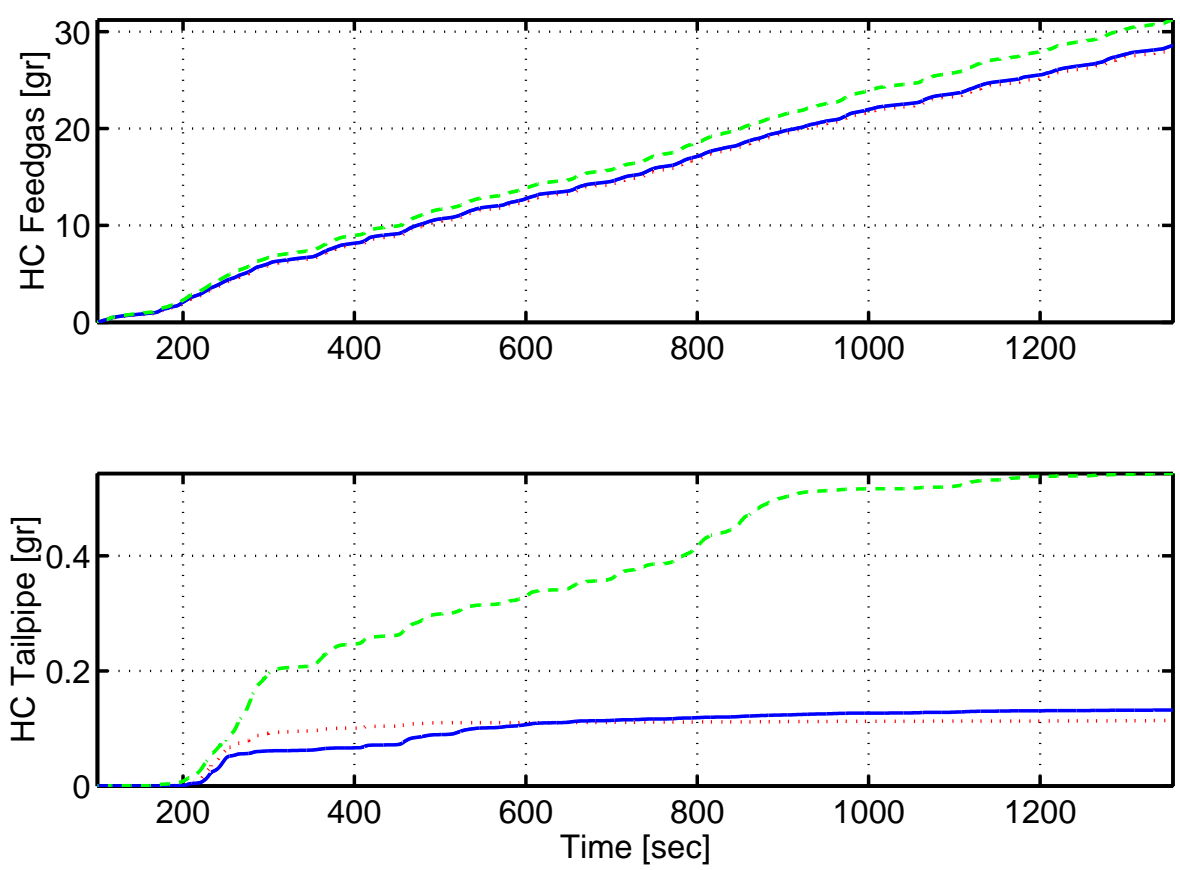

Fig. 27. 2.3L Engine. Pre- and Post-catalyst cumulative $H C$ emissions in grams at brick two. The dotted (red) refers to the controller with tailpipe $A / F$ measured at Brick 1 , solid line (blue) refers to the controller with tailpipe $A / F$ measured at Brick 2 and the dot-dashed line (green) refers to the baseline controller.
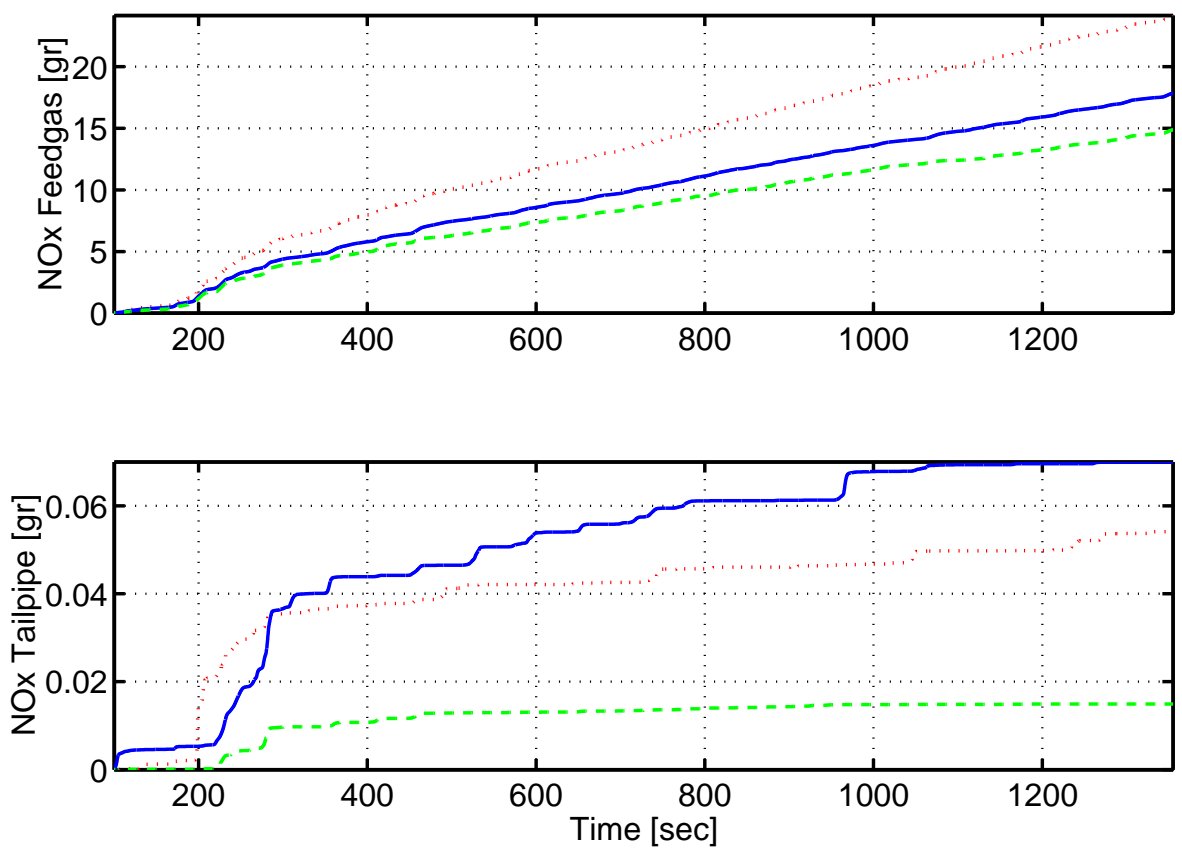

Fig. 28. 2.3L Engine. Pre- and Post-catalyst cumulative $N O x$ emissions in grams at brick two. The dotted (red) refers to the controller with tailpipe $A / F$ measured at Brick 1 , solid line (blue) refers to the controller with tailpipe $A / F$ measured at Brick 2 and the dot-dashed line (green) refers to the baseline controller. 